\title{
Felicidad organizacional: mediación entre la valoración del empleado y la productividad. Una mirada a su gestión en el sector estatal ${ }^{\star}$
}

\author{
Ana Lorena Malluk Marenco** \\ Recibido: 2018-01-21. Enviado a pares: 2018-01-25. \\ Aprobado por pares: 2018-02-25. Aceptado: 2018-02-28 \\ https://doi.org/10.22395/angr.v17n33a10
}

\begin{abstract}
Resumen
El artículo aborda el tema de la felicidad organizacional y su incidencia en la relación empleado-productividad. El objetivo de la investigación es diagnosticar el estado de la percepción de felicidad organizacional de los administrativos de una institución educativa superior de carácter estatal del departamento de Córdoba, Colombia, a partir de las dimensiones: trabajo en equipo, satisfacción, compromiso e innovación. Mediante una investigación cuali-cuantitativa de corte exploratorio, se planteó una muestra representativa y aleatoria constituida por 297 empleados administrativos adscritos a tres sedes ubicadas en los municipios de Montería, Lorica y Berástegui, con un margen de error de 0,03\%.

Los hallazgos evidencian el potencial de las dimensiones trabajo en equipo y compromiso con la organización/función y la necesidad de fortalecer la satisfacción laboral e innovación como factores determinantes en las expectativas y experiencias individuales, las relaciones interpersonales, la motivación, la capacidad de adaptación, la diversidad de métodos, el bienestar laboral y la apropiación.
\end{abstract}

Se concluye que la universidad -caracterizada por ser tradicional e influyente en el medio, con una estructura compleja, con un número de empleados superior a seiscientas personas y sujeta a vigilancia por parte del Estado- ha realizado esfuerzos en materia de clima laboral, pero carece de políticas institucionales que dimensionen la felicidad organizacional como el engranaje para el desarrollo empresarial desde la valoración del empleado y la productividad.

Palabras clave: compromiso, innovación, satisfacción, trabajo en equipo, desarrollo organizacional, productividad, universidad.

El texto es resultado del proyecto de investigación Estado actual de la percepción de felicidad organizacional de los administrativos de una institución educativa superior de carácter oficial de Montería, Colombia, desarrollado por Linda Lucía Pernett Rodríguez, María Alejandra Ponce Cárdenas y María Fernanda Carrascal Piñeres. Semillero Gestores de la Comunicación GECO, grupo Comunicación, Derecho y Humanidades Coedu. Universidad Pontificia Bolivariana.

. Docente del programa de Comunicación Social-Periodismo, Universidad Pontificia Bolivariana, seccional Montería, magíster en Comunicación, coordinadora semillero GECO, investigadora del grupo Coedu. ana.malluk@upb.edu.co. Orcid: https://orcid.org/0000-0003-1049-4078 


\title{
Organizational happiness: mediation between employee valuation and productivity. A look at its management in the state sector
}

\begin{abstract}
This paper addresses the issue of organizational happiness and its impact on the employee-productivity relationship. The goal of the research was to diagnose the state of the perception of organizational happiness of the administrative personnel of a public higher education institution Department of Córdoba (Colombia), relying on four dimensions: Teamwork, satisfaction, commitment, and innovation. Through a qualitative-quantitative and exploratory research, a representative and random sample comprised by 297 administrative employees assigned to three offices located in the municipalities of Montería, Lorica and Berástegui, with a margin of error of $0.03 \%$, was analyzed. The findings show the potential of the teamwork and commitment dimensions to the organization / function, and the need to strengthen job satisfaction and innovation as determining factors in individual expectations and experiences, interpersonal relationships, motivation, adaptability, diversity of methods, work well-being, and appropriation.

It is concluded that the university -distinguished by being traditional and influential, with a complex structure, a number of employees that exceeds six hundred people, and subject to surveillance by the State- has made efforts in matters of work climate, but lacks institutional policies that measure organizational happiness as the gear for business development based on employee assessment and productivity.
\end{abstract}

Keywords: Commitment, innovation, satisfaction, teamwork, organizational development, productivity, university.

\section{Felicidade organizacional: mediação entre a valorização do empregado e a produtividade. Um olhar sobre a sua gestão no setor estatal}

\section{Resumo}

O artigo aborda o tema da felicidade organizacional e sua incidência na relação empregado-produtividade. O objetivo da pesquisa é diagnosticar o estado da percepção de felicidade organizacional dos funcionários administrativos de uma instituição educativa superior de caráter estatal do departamento de Córdoba, Colômbia, a partir das dimensões: trabalho em equipe, satisfação, compromisso e inovação. Mediante uma pesquisa qualitativa-quantitativa de corte exploratório, propôs-se uma amostra representativa e aleatória constituída por 297 empregados administrativos adscritos a três sedes localizadas nos municípios de Montería, Lorica e Berástegui, com uma margem de erro de 0,03 \%.

As descobertas evidenciam o potencial das dimensões trabalho em equipe e compromisso com a organização/função e a necessidade de fortalecer a satisfação no trabalho e inovação como fatores determinantes nas expectativas e experiências individuais, as relações interpessoais, a motivação, a capacidade de adaptação, a diversidade de métodos, o bem-estar trabalhista e a apropriação.

Conclui-se que a universidade -caracterizada por ser tradicional e influente no meio, com uma estrutura complexa, com um número de empregados superior a seiscentas pessoas e sujeita à vigilância por parte do Estado- tem realizado esforços em matéria de clima trabalhista, mas carece de políticas institucionais que dimensionem a felicidade organizacional como a engrenagem para o desenvolvimento empresarial a partir da valorização do empregado e da produtividade.

Palavras-chave: compromisso, inovação, satisfação, trabalho em equipe, desenvolvimento organizacional, produtividade, universidade. 


\section{Introducción}

Desde postulados tradicionales, las organizaciones se conciben como un grupo humano que combina conocimientos individuales y conocimientos especializados para crear valor en conjunto (Drucker, 1993). Son estructuras sistemáticas constituidas por personas que comparten políticas y normas, se jerarquizan por mandos, establecen un propósito común y coordinan funciones para alcanzar objetivos productivos, mediante el desarrollo de tareas organizadas (Betancourt, 1985). En sentido más amplio, las profundas y continuas transformaciones que experimenta la sociedad actual, han influenciado el quehacer de las organizaciones sumando un nuevo componente: la felicidad. De esta manera, la productividad deja de ser el elemento protagónico de la gestión y la felicidad se dimensiona más allá de una emoción natural humana (Dutschke, 2013).

Para Caycho (2010), la felicidad es un estado emocional generado por la interacción de diferentes condiciones que actúan sobre el individuo, lo que provoca respuestas con efectos positivos en múltiples ámbitos de la vida, favoreciendo así, el mayor desarrollo social y económico a través de la autorrealización con el trabajo (Carballeira, González y Marrero, 2015). Las personas con altos niveles de autoestima presentan conductas saludables y optimismo (Rodríguez y Caño, 2012), lo que se traduce en estados emocionales positivos, aprovechamiento de fortalezas y capacidades, calidad de vida física y mental (Quiceno y Vinaccia, 2012), sensación de realización personal (Gutiérrez y Gonçalves, 2013) y adaptación al entorno (Oberle, Schonert-Reichl y Zumbo, 2011).

Sin embargo, aunque hay una apuesta interesante en el tema, existen aún contextos laborales donde predominan estados emocionales asociados a la depresión, el estrés y el pesimismo, problemas de salud mental, dificultades para la integración social, bajo autoconcepto y niveles de autoeficacia deficitarios (Pulido y Herrera, 2018) que sin duda afectan negativamente la gestión empresarial.

Los avances en cuanto al enfoque de la psicología positiva de Martín Seligman plantean el estudio científico del funcionamiento humano óptimo desde el abordaje del optimismo y la atención a las fuentes de la salud psicológica, social y organizacional. Así, el tema empieza a permear el mundo de las organizaciones, más aún, porque enfatiza en el optimismo y en el funcionamiento humano positivo en lugar de centrarse en la psicopatología y la disfunción, cuya meta no es remediar los déficits, sino impulsar las fortalezas para comprender y facilitar la felicidad y el bienestar psicológico (Seligman, 1999).

Las investigaciones llevadas a cabo conducen a descubrimientos que sugieren cambios desde diferentes dimensiones: la psicología no solo se preocupa por solucionar únicamente las cosas que van mal en la vida, también por construir cualidades positivas (Seligman y Csikszentmihalyi, 2000), la gestión se enfoca a consolidar una empresa competitiva que sobreviva en el medio potencializando la organización saludable y positiva; y la comunicación se articula con los procesos participativos que favorecen el diálogo, las relaciones interpersonales, los flujos de información, el bienestar general y la satisfacción de los grupos de interés. 
De esta manera emerge la psicología organizacional positiva, entendida como el estudio científico del funcionamiento óptimo de la salud de las personas y de los grupos en las organizaciones y como la gestión efectiva del bienestar psicosocial en el trabajo y del desarrollo de las organizaciones saludables (Salanova, Martínez, y Llorens, 2005; 2014). Bajo esta mirada, se empiezan a descubrir las características que configuran una vida organizacional plena, a través de la búsqueda de emociones positivas y del desarrollo de aptitudes para multiplicarlas, el empleo de las propias fortalezas para obtener gratificaciones auténticas y el poner las fortalezas personales al servicio de algo que trascienda (Fernández, 2015).

El auge a nivel mundial provoca que en 2012 la Asamblea General de Naciones Unidas proclame el Día Internacional de la Felicidad:

Es necesario que los Estados miembros elaboren nuevas medidas para reflejar la búsqueda de la felicidad y el bienestar en el desarrollo del planeta, con miras a orientar políticas públicas, teniendo en cuenta: 1. Conciencia de que la búsqueda de la felicidad es un objetivo humano fundamental. 2. Reconocer la pertinencia de la felicidad y del bienestar como objetivos y aspiraciones universales en la vida de los seres humanos de todo el mundo y la importancia de que se reconozcan en los objetivos de las políticas públicas. 3. Necesidad de que se aplique al crecimiento económico un enfoque más inclusivo, equitativo y equilibrado que promueva el desarrollo sostenible, la erradicación de la pobreza y la búsqueda de la felicidad y el bienestar de todos los pueblos. (Citado por Fernández, 2015, pp. 12-35).

El concepto de felicidad productiva se fortalece. Las investigaciones llevadas a cabo en Madrid profundizan sobre el capital emocional como palanca personal y organizativa que incide en la productividad, cuya clave está en la combinación de la gestión de las operaciones -eficiencia operativa-y la gestión de los individuos -felicidad profesional-. No obstante, hallazgos científicos en Inglaterra demuestran que la felicidad de los trabajadores no sólo tiene un efecto directo en la productividad -ayudando a aumentarla en un $12 \%$ - sino también que contribuye a mejorar los resultados de las empresas (Oswald, Proto y Sgroi, 2015).

En Portugal se aborda la relación felicidad-remuneración y se concluye que la remuneración no es el factor más determinante para que la persona se sienta feliz en la empresa: son más importantes el reconocimiento, la posibilidad de desarrollo personal y profesional, y el ambiente interno (Dutschke, 2013). Posteriormente, se avanza en el modelo de organizaciones felices en las que cada individuo, en todos los niveles jerárquicos, tiene fortalezas, trabaja en equipo hacia un objetivo común, obtiene satisfacción al desarrollar nuevos productos/servicios y, a través de estos, suele proporcionar una diferencia positiva en la vida de otros (Dutschke, 2013).

La felicidad considera dimensiones como la participación de los profesionales con la organización y la función, la satisfacción con el trabajo y el compromiso positivo con la organización y la función (Fisher, 2010). Asimismo, se destaca el compromiso afectivo 
con la organización, el bienestar en la función y la satisfacción en el trabajo (Hosie, Cooper y Sevastos, 2007), siendo esta última una variable central en cualquier modelo que pretenda explicar consecuencias organizacionalmente relevantes (Salessi y Omar, 2017).

Otras fuentes indican que el $25 \%$ del éxito laboral depende del coeficiente intelectual y que el $75 \%$ restante se relaciona con el estado anímico y con la forma como el cerebro se conecta con las demás personas, por lo tanto, la felicidad aumenta el rendimiento laboral hasta en un 33 \% (Achor, 2010). Las personas felices tienen la posibilidad de desempeñarse en su rango alto de capacidades, liberan endorfinas y dopaminas en el cerebro, lo que les permite pensar mejor, más rápido, ser más creativos y asertivos (Revista Líderes, 2014).

Las discusiones en torno al tema conducen a concluir que la carencia de trabajo en equipo, satisfacción, compromiso e innovación entre el recurso humano provoca falta de experiencias felices. Las habilidades de desempeño blandas se ven afectadas por el estado emocional del trabajador: si el buen funcionamiento de las organizaciones exige un óptimo clima laboral, entonces la felicidad de los empleados es determinante para la motivación y viceversa. Por lo tanto, es necesario gestionar el recurso humano para avanzar en actitudes positivas, buenas relaciones con los demás, establecimiento de metas, desarrollo y apropiación de las acciones encomendadas y otros valores en el contexto laboral (Tarragona, 2016).

En Argentina, las investigaciones evidencian la relación entre la cultura y los valores en el trabajo, especialmente en lo que respecta a la comunicación del empleado con el superior y al bienestar. Los hallazgos demuestran que, en el ámbito organizacional, los patrones asociados a las normas y valores de cada persona aportan al desarrollo de la empresa en cuanto a la conservación (valores que los llevan al mantenimiento de las tradiciones, costumbres, al respeto de las leyes y reglamentos); la autotrascendencia (cuidado y preocupación por el bienestar de las personas cercanas), la autopromoción (logro de objetivos personales y ejercicio de la autoridad mediante la conducción de grupos de trabajo) y la apertura al cambio (independencia del pensamiento, innovación y poder resolver problemas habituales con metodologías alternativas (Nader y Castro, 2010).

Independientemente del carácter jurídico, los esfuerzos que realizan las organizaciones tienden al bienestar de las personas y al mejoramiento continuo de los procesos, más aún cuando el individuo es considerado el activo más importante con que cuentan y pieza clave para alcanzar un nivel óptimo de desarrollo. A diferencia de las empresas privadas -cuyas normas y políticas son autónomas y propenden por la satisfacción de necesidades particulares que se traducen en las intenciones de los inversionistas en el producto/servicio para lucrarse y controlar el capital (Cardona, 2002)-; las entidades estatales están sujetas a la influencia directa o indirecta de los poderes públicos, en razón de la propiedad y de la participación financiera o de las normas que la rigen, lo que las impregna de condiciones particulares asociadas a la toma de decisiones, rendimiento/ productividad, compromiso con la organización, sentido de pertenencia, proactividad, recursividad, satisfacción personal, estabilidad laboral, innovación, entre otros. 
A pesar de que el talento humano es considerado un activo fundamental, pieza clave para lograr las metas, existen organizaciones que no enfocan su gestión hacia el compromiso y motivación para alcanzar el éxito, algunas siguen cometiendo errores obviando la situación en tiempos donde las tendencias evidencian cambios estructurales en el estilo de liderazgo y en la gerencia del personal la cual, necesariamente, se relaciona con el bienestar de los empleados.

Las instituciones de educación superior no son ajenas a esta realidad, esto porque la falta de compromiso y motivación en los funcionarios afecta a la sociedad en su conjunto por la trascendencia del servicio que ofertan, por el número de beneficiarios, por el nivel de relacionamiento y por la incidencia en la transformación de todo un colectivo. La universidad objeto de estudio, institución estatal fundada en 1962 en Montería, Colombia, comprende la planificación, supervisión y ejecución directa de planes de estudio y educación escolarizada en el nivel superior académico, ofrece programas profesionales, tecnológicos y de posgrados en las modalidades presencial y a distancia. Es una organización tradicional, influyente en el medio, con una estructura compleja por el servicio que presta y por las funciones sustantivas que la constituyen: docencia, investigación y proyección; con un número de empleados superior a 600 y sujeta a vigilancia por parte del Estado.

Al igual que toda organización, esta universidad requiere de directivos con capacidad de liderazgo que dimensionen la felicidad como un comportamiento organizacional, resultado de una visión estratégica (Dutschke, 2013) y de trabajadores motivados y comprometidos con la construcción de una institución competitiva: individuos felices y profesionales apropiados, a los que se le reconocen sus talentos y valoran las particularidades, más aún porque, el contar con empleados felices, contribuye a mantener buenas relaciones interpersonales, al mejoramiento continuo, al aumento y calidad en la productividad, al óptimo desarrollo de los procesos educativos, al adecuado manejo de los recursos y a la efectiva gestión administrativa de acuerdo a la normatividad. Todo ello garantiza la existencia de un funcionario feliz que pueda desempeñarse con la totalidad de sus capacidades para garantizar la oportuna gestión del sistema educativo.

El hecho de saber que la felicidad incide directamente en la gestión del recurso humano, es producto de un comportamiento organizacional que trasciende las emociones y es resultado de la visión estratégica y de conocer que la universidad objeto de estudio tiene enorme trascendencia en el departamento de Córdoba -lo que se convierte en un referente que ilustra un fenómeno poco abordado en este contexto-, motiva a indagar bajo un enfoque cuali-cuantitativo el estado actual de la percepción de felicidad de un grupo de empleados para crear el escenario propicio hacia la gestión de felicidad organizacional (Fernández, 2015), haciendo uso de técnicas de información como el análisis documental, la observación no participante y la encuesta.

Lo anterior conduce a formular la siguiente pregunta de investigación ¿Cuál es el estado actual de la percepción de felicidad organizacional de los empleados administrativos de una institución educativa superior de carácter estatal del departamento de Córdoba, 
Colombia, a partir de las dimensiones: trabajo en equipo, satisfacción, compromiso e innovación?

\section{Metodología}

La investigación es de enfoque cuali-cuantitativa, estudia la percepción de felicidad de los funcionarios administrativos de la universidad objeto de estudio en su contexto natural, sacando e interpretando fenómenos de acuerdo con las opiniones de las personas implicadas (Stake, 2007) y analizando estadísticamente los datos obtenidos para verificar, aprobar o rechazar las relaciones entre las variables definidas operacionalmente (Rodríguez, 2010).

En cuanto al método, se empleó el estudio de caso para comprender de manera profunda la realidad del objeto de estudio que, aunque no generaliza el fenómeno, sí lo ilustra, lo que se convierte en un importante referente de investigación. El método aportó la posibilidad de abordar el fenómeno contemporáneo dentro de su contexto real de existencia, cuando los límites entre ambos no son claramente evidentes y en los cuales existen múltiples fuentes de evidencia que pueden usarse (Yin, 1993) para abordar el problema desde diferentes dimensiones.

El alcance es de tipo exploratorio (Dankhe, 1986), examina el fenómeno en una ins titución del sector estatal para aumentar el grado de familiaridad y conocimiento sobre aspectos relativamente desconocidos en el contexto de Montería, Colombia; investiga problemas del comportamiento humano considerados cruciales para determinada área y permite identificar variables/conceptos que puedan servir para investigaciones posteriores ofreciendo postulados verificables (Hernández Sampieri, Fernández y Baptista, 2006).

La población objetivo la constituye la comunidad administrativa, distribuida en tres sedes ubicadas en los municipios de Montería, Lorica y Berástegui. El total de los administrativos es de 443 personas. Para establecer el diseño de muestreo se tuvo en cuenta la homogeneidad de las características de la población teniendo en cuenta el tipo de variables del instrumento, se utilizó la fórmula propuesta por Särndal, Swensson y Wretman (2003) para estimar proporciones cuando se conoce el tamaño de la población total, que para el presente caso es $\mathrm{N}=443$.

El número de encuestas a realizar se expresa en la siguiente ecuación:

Donde

Z: corresponde al valor tabulado de la distribución normal.

P: la proporción que hace máximo el tamaño muestral.

e: error muestral.

Con una confianza del $95 \%$, se obtuvo un valor tabulado de la distribución normal de 1,96 y se utilizó el valor de proporción de éxitos de P = 0,5 (este valor maximiza el tamaño de muestra final). Es decir, utilizando un error muestral del 3 \% se tuvo que: 
Así, con una confianza del $95 \%$, el número de encuestas analizadas corresponden a 297 funcionarios. Luego, aplicando el mecanismo de selección de coordinado negativo se procedió a seleccionar los funcionarios que tuvieran los 297 valores aleatorios menores, de esta manera, el tamaño de la muestra no determina lo que quiere medir el instrumento, este es solo uno de los factores para analizar la validez de la investigación teniendo en cuenta una población determinada. Finalmente, se estableció que el tamaño de la muestra aleatoria simple cuenta con un margen de error del 0,03\%, que equivale a 297 administrativos de 90 dependencias, siendo los cargos desempeñados el principal criterio de selección de la muestra.

Teniendo en cuenta las variables del instrumento, se realizó la validación estadística respectiva, luego se cruzaron los resultados de cada variable mediante las pruebas de independencia Chi-Cuadrado de Pearson y finalmente se hizo un análisis multivariado a través de un análisis de correspondencias múltiples (ACM).

En cuanto a las técnicas de recolección de información, se empleó el análisis documental para estructurar el estudio. El análisis de los lineamientos corporativos de la universidad permitió establecer cómo desde las políticas se conciben y desarrollan aspectos asociados a las variables trabajo en equipo, satisfacción, compromiso e innovación en el entorno organizacional. También se indagó sobre la existencia o carencia de estudios de clima organizacional, bienestar laboral o afines. Asimismo, el estudio se complementó con el test CLA Clima Laboral, para evaluar el clima laboral de las empresas y las organizaciones ( Corral y Pereña, 2003) y con el diagnóstico de comunicación organizacional de la institución educativa Cristóbal Colón de Montería, Colombia (Malluk y Bedoya, 2016) que ofreció un cuestionario validado que sirvió de referente para el diseño de la encuesta.

Otra técnica de recolección de información empleada fue la encuesta dirigida a administrativos, sirvió para realizar comparaciones entre áreas de trabajo en cada variable. Finalmente, su uso permitió determinar la influencia del área de trabajo en la percepción sobre felicidad organizacional y establecer asociaciones mediante un análisis multivariado a través del análisis de correspondencias múltiples. Se diseñó y aplicó un instrumento tipo escala de Likert que contó con 39 ítems distribuidos en cuatro variables; se formularon 39 proposiciones utilizando en la redacción expresiones claras, un lenguaje al alcance de toda la muestra y evitando tecnicismos. En dicha escala las opciones de respuesta fueron: nunca (1), rara vez (2), usualmente (3) y siempre (4). La encuesta se sometió a juicio de expertos para el ajuste de la redacción y comprensión de las preguntas, un comunicador social experto en gestión de la comunicación en organizaciones y un psicólogo especialista en gestión del talento humano.

Por último, la observación no participante fue otra técnica de recolección de información que complementó el proceso de aplicación de la encuesta, gracias a esto se pudo establecer el contacto con los administrativos al momento de aplicar el instrumento y 
las impresiones relevantes del investigador con respecto a las reacciones del personal; aspectos claves para describir e interpretar el contexto organizacional y para conocer el reconocimiento de la población con respecto al proceso de diagnóstico, de sus resultados e impacto.

Con base en el problema, pregunta y objetivos, se identificaron cuatro variables: trabajo en equipo, satisfacción en el trabajo, compromiso e innovación/creatividad (tabla 1).

Tabla 1. Variables, subvariables y postulados teóricos

\begin{tabular}{|c|c|c|}
\hline Variables & Postulado teórico & Subvariables \\
\hline $\begin{array}{l}\text { Trabajo en } \\
\text { equipo }\end{array}$ & $\begin{array}{l}\text { Capacidad para transformar posibilidades en soluciones } \\
\text { reales que contribuyan a la sostenibilidad de la organi- } \\
\text { zación (Dutschke, 2013). }\end{array}$ & $\begin{array}{l}\text { Capacidades } \\
\text { intelectuales } \\
\text { Respeto } \\
\text { Colaboración }\end{array}$ \\
\hline $\begin{array}{l}\text { Satisfacción } \\
\text { en el trabajo }\end{array}$ & $\begin{array}{l}\text { Perspectiva favorable o desfavorable de los trabajadores } \\
\text { sobre su trabajo expresado a través del grado de con- } \\
\text { cordancia que existe entre las expectativas con respecto } \\
\text { al trabajo, las recompensas que le ofrece, las relaciones } \\
\text { interpersonales y el estilo gerencial (Gamboa, 2006). }\end{array}$ & $\begin{array}{l}\text { Experiencia } \\
\text { Expectativas } \\
\text { Relaciones } \\
\text { interpersonales }\end{array}$ \\
\hline Compromiso & $\begin{array}{l}\text { Decisión personal desde la voluntad que nace del vínculo } \\
\text { emocional, se enriquece desde la norma y se expresa a } \\
\text { través de la pertenencia a partir de las facetas: 1. Com- } \\
\text { promiso desde lo emocional: grado de identificación } \\
\text { desarrollado por los trabajadores cuando satisface las } \\
\text { necesidades individuales y cubre las expectativas labo- } \\
\text { rales. 2. Compromiso normativo: se fundamenta en las } \\
\text { reglas recíprocas institucionales; en la consolidación } \\
\text { de las normas que beneficien la empresa y representen } \\
\text { los intereses de los trabajadores. 3. Compromiso desde } \\
\text { la pertenencia: indica la necesidad de los empleados } \\
\text { de continuar en el puesto de trabajo, motivado por la } \\
\text { satisfacción personal, el sentido de identificación con la } \\
\text { empresa y la remuneración que recibe a cambio de las } \\
\text { funciones que desempeña (Meyer y Allen, 1984). }\end{array}$ & $\begin{array}{l}\text { Emocional } \\
\text { Normativo } \\
\text { Pertenencia }\end{array}$ \\
\hline Innovación & $\begin{array}{l}\text { Capacidad de convertir las ideas en nuevos y mejorados } \\
\text { procesos, servicios y productos que el mercado nece- } \\
\text { site, reconozca y valore por su aporte. Capacidad para } \\
\text { adaptarse a nuevas situaciones y factores del entorno } \\
\text { (Shona, Brown y Eisenhardt, 1997) y desprenderse de } \\
\text { conocimientos y normas obsoletas, así como de las } \\
\text { barreras que impiden la adquisición y el procesamiento } \\
\text { de nueva información (Baker y Sinkula, 1999). }\end{array}$ & $\begin{array}{l}\text { Cultura organizacional } \\
\text { Motivación } \\
\text { Creatividad }\end{array}$ \\
\hline
\end{tabular}




\section{Resultados}

La validación estadística de la encuesta se realizó a través de los coeficientes de confiabilidad alfa de Cronbach y Guttman. Según el método alfa de Cronbach el 82 \% corresponde a la probabilidad de que los encuestados comprendieron las preguntas. Los coeficientes de Guttman arrojan en promedio una probabilidad aproximada del $80 \%$, indicando que el instrumento es viable y fue comprendido; por lo que existe consistencia interna de los ítems (tabla 2).

Tabla 2. Cronbach. Estadísticos de fiabilidad

\begin{tabular}{|c|c|}
\hline \multicolumn{2}{|c|}{ Cronbach. Estadísticos de fiabilidad } \\
\hline Alfa de Cronbach & N. ${ }^{\circ}$ elementos \\
\hline 0,817 & 39 \\
\hline
\end{tabular}

\begin{tabular}{|c|c|c|}
\hline \multicolumn{3}{|c|}{ Guttman. Estadísticos de fiabilidad } \\
\hline \multirow{3}{*}{ Lambda } & 1 & 0,796 \\
\cline { 2 - 3 } & 2 & 0,838 \\
\cline { 2 - 3 } & 3 & 0,817 \\
\cline { 2 - 3 } & 4 & 0,694 \\
\cline { 2 - 3 } & 5 & 0,817 \\
\hline \multicolumn{2}{|c|}{ No. elementos } & 39 \\
\hline
\end{tabular}

Fuente: elaboración propia

La estructura de los resultados se presenta por variables a partir de la Prueba de Independencia Chi-cuadrado de Pearson. Se observa la percepción por tipo de vinculación para identificar la diferencia estadística o dependencia.

\section{Variable 1. Trabajo en equipo}

Tabla 3. Solicitud de opiniones, ideas y sugerencias en forma regular para la toma de decisiones por parte del jefe

\begin{tabular}{|c|l|c|c|c|}
\cline { 3 - 5 } \multicolumn{2}{c|}{} & Frecuencia & $\%$ & \% acumulado \\
\hline \multirow{4}{*}{ Opciones de respuesta } & Nunca & 21 & 7,1 & 7,1 \\
\cline { 2 - 5 } & Rara vez & 41 & 13,8 & 20,9 \\
\cline { 2 - 5 } & Usualmente & 121 & 40,7 & 100,0 \\
\cline { 2 - 5 } & Siempre & 114 & 38,4 & 59,3 \\
\cline { 2 - 5 } & Total & 297 & 100,0 & \\
\hline
\end{tabular}

Fuente: elaboración propia, 2017. 
Felicidad organizacional: mediación entre la valoración del empleado y la productividad. Una mirada ...

Tabla 4. Solicitud de opiniones, ideas y sugerencias en forma regular para la toma de decisiones por parte del jefe según cargo ocupado

\begin{tabular}{|c|c|c|c|c|c|c|}
\cline { 2 - 7 } \multicolumn{2}{c|}{} & Nunca & Rara vez & Siempre & Usualmente & Total \\
\hline \multirow{4}{*}{ Cargo } & Auxiliar & 3 & 5 & 12 & 14 & 34 \\
\cline { 2 - 7 } & Jefe & 1 & 1 & 13 & 8 & 23 \\
\cline { 2 - 7 } & Profesional & 7 & 18 & 46 & 44 & 115 \\
\cline { 2 - 7 } & Secretario & 3 & 6 & 11 & 20 & 40 \\
\cline { 2 - 7 } & Técnico & 7 & 11 & 32 & 35 & 85 \\
\hline \multicolumn{2}{|c|}{ Total } & 21 & 41 & 114 & 121 & 297 \\
\hline
\end{tabular}

\begin{tabular}{|l|c|c|c|}
\hline \multicolumn{1}{|c|}{ Prueba de independencia } & Valor & Gl & Sig. asintótica (bilateral) \\
\hline Chi-cuadrado de Pearson & $7,203^{\text {a }}$ & 12 & 0,844 \\
\hline Razón de verosimilitudes & 7,609 & 12 & 0,815 \\
\hline N. ${ }^{\circ}$ de casos válidos & 297 & & \\
\hline
\end{tabular}

Fuente: elaboración propia

Pvalor $=0.844>0.05$. Se acepta la hipótesis de independencia entre las variables: la opinión de los administrativos es independiente del tipo de cargo que ocupe.

Tabla 5. Disposición del compañero para colaborar en caso de que se requiera

\begin{tabular}{|l|l|c|c|c|}
\cline { 2 - 5 } \multicolumn{2}{c|}{} & Frecuencia & $\%$ & \% acumulado \\
\hline \multirow{4}{*}{ Opciones de respuesta } & Nunca & 2 & 0,7 & 0,7 \\
\cline { 2 - 5 } & Rara vez & 20 & 6,7 & 7,4 \\
\cline { 2 - 5 } & Usualmente & 143 & 48,1 & 100,0 \\
\cline { 2 - 5 } & Siempre & 132 & 44,4 & 51,9 \\
\cline { 2 - 5 } & Total & 297 & 100,0 & \multicolumn{1}{c}{} \\
\hline
\end{tabular}

Fuente: elaboración propia

Tabla 6. Disposición del compañero para colaborar en caso de que se requiera según cargo ocupado

\begin{tabular}{|l|l|c|c|c|c|c|}
\cline { 3 - 7 } \multicolumn{2}{c|}{} & Nunca & Rara vez & Siempre & Usualmente & Total \\
\hline \multirow{4}{*}{ Cargo } & Auxiliar & 1 & 1 & 16 & 16 & 34 \\
\cline { 2 - 7 } & Jefe & 0 & 0 & 15 & 8 & 23 \\
\cline { 2 - 7 } & Profesional & 0 & 11 & 43 & 61 & 115 \\
\cline { 2 - 7 } & Secretario & 0 & 3 & 24 & 13 & 40 \\
\cline { 2 - 7 } & Técnico & 1 & 5 & 34 & 45 & 85 \\
\hline \multirow{4}{*}{ Total } & 2 & 20 & 132 & 143 & 297 \\
\hline
\end{tabular}




\begin{tabular}{|l|c|c|c|}
\hline \multicolumn{1}{|r|}{ Prueba de independencia } & Valor & Gl & Sig. asintótica (bilateral) \\
\hline Chi-cuadrado de Pearson & $17,88^{7 a}$ & 12 & 0,119 \\
\hline Razón de verosimilitudes & 19,336 & 12 & 0,081 \\
\hline N. ${ }^{\circ}$ de casos válidos & 297 & & \\
\hline
\end{tabular}

Fuente: elaboración propia

Pvalor $=0.119>0.05$. Se acepta la hipótesis de independencia entre las variables: la opinión de los administrativos es independiente del tipo de cargo que ocupe.

Tabla 7. Disposición personal para colaborarle al compañero en caso de que lo necesite

\begin{tabular}{|c|l|c|c|c|}
\cline { 3 - 5 } \multicolumn{2}{c|}{} & Frecuencia & $\%$ & \% acumulado \\
\hline \multirow{3}{*}{$\begin{array}{c}\text { Opciones de } \\
\text { respuesta }\end{array}$} & Rara vez & 8 & 2,7 & 2,7 \\
\cline { 2 - 5 } & Usualmente & 107 & 36,0 & 100,0 \\
\cline { 2 - 5 } & Siempre & 182 & 61,3 & 64,0 \\
\cline { 2 - 5 } & Total & 297 & 100,0 & \\
\hline
\end{tabular}

Fuente: elaboración propia

Tabla 8. Disposición personal de los administrativos para colaborarle al compañero en caso de que lo necesite

\begin{tabular}{|c|l|c|c|c|c|c|}
\cline { 3 - 7 } \multicolumn{2}{c|}{} & Nunca & Rara vez & Siempre & Usualmente & Total \\
\hline \multirow{4}{*}{ Cargo } & Auxiliar & & 3 & 20 & 11 & 34 \\
\cline { 2 - 7 } & Jefe & & 0 & 18 & 5 & 23 \\
\cline { 2 - 7 } & Profesional & & 3 & 74 & 38 & 115 \\
\cline { 2 - 7 } & Secretario & & 1 & 27 & 12 & 40 \\
\cline { 2 - 7 } & Técnico & & 1 & 43 & 41 & 85 \\
\hline \multirow{7}{*}{ Total } & & 8 & 182 & 107 & 297 \\
\hline
\end{tabular}

\begin{tabular}{|l|c|c|c|}
\hline \multicolumn{1}{|c|}{ Prueba de independencia } & Valor & Gl & $\begin{array}{c}\text { Sig. Asintótica (bila- } \\
\text { teral) }\end{array}$ \\
\hline Chi-cuadrado de Pearson & $14,863^{\text {a }}$ & 8 & 0,062 \\
\hline Razón de verosimilitudes & 13,803 & 8 & 0,087 \\
\hline N. ${ }^{\circ}$ de casos válidos & 297 & & \\
\hline
\end{tabular}

Fuente: elaboración propia

Pvalor $=0,062>0,05$. Se acepta la hipótesis de independencia entre las variables: la opinión de los administrativos es independiente del tipo de cargo que ocupe. 
Felicidad organizacional: mediación entre la valoración del empleado y la productividad. Una mirada ...

Tabla 9. Alta tramitología y exceso de personal en la organización

\begin{tabular}{|c|c|c|c|c|}
\cline { 3 - 5 } \multicolumn{1}{c|}{} & Frecuencia & $\%$ & \% acumulado \\
\hline \multirow{4}{*}{$\begin{array}{c}\text { Opciones de } \\
\text { respuesta }\end{array}$} & Nunca & 25 & 8,4 & 8,4 \\
\cline { 2 - 5 } & Rara vez & 88 & 29,6 & 38,0 \\
\cline { 2 - 5 } & Usualmente & 119 & 40,1 & 100,0 \\
\cline { 2 - 5 } & Siempre & 65 & 21,9 & 59,9 \\
\cline { 2 - 5 } & Total & 297 & 100,0 & \\
\hline
\end{tabular}

Fuente: elaboración propia

Tabla 10. Alta tramitología y exceso de personal en la organización según cargo ocupado

\begin{tabular}{|c|l|c|c|c|c|c|}
\cline { 3 - 7 } \multicolumn{2}{c|}{} & Nunca & Rara vez & Siempre & Usualmente & Total \\
\hline \multirow{5}{*}{ Cargo } & Auxiliar & 3 & 12 & 8 & 11 & 34 \\
\cline { 2 - 7 } & Jefe & 6 & 6 & 7 & 4 & 23 \\
\cline { 2 - 7 } & Profesional & 7 & 29 & 22 & 57 & 115 \\
\cline { 2 - 7 } & Secretario & 2 & 15 & 4 & 19 & 40 \\
\cline { 2 - 7 } & Técnico & 7 & 26 & 24 & 28 & 85 \\
\hline \multirow{7}{*}{ Total } & 25 & 88 & 65 & 119 & 297 \\
\hline
\end{tabular}

\begin{tabular}{|l|c|c|c|}
\hline \multicolumn{1}{|c|}{ Prueba de independencia } & Valor & Gl & Sig. asintótica (bilateral) \\
\hline Chi-cuadrado de Pearson & $24,953^{\mathrm{a}}$ & 12 & 0,015 \\
\hline Razón de verosimilitudes & 23,155 & 12 & 0,026 \\
\hline N. ${ }^{\circ}$ de casos válidos & 297 & & \\
\hline
\end{tabular}

Fuente: elaboración propia

Pvalor $=0,015<0,05$. Se rechaza la hipótesis de independencia entre las variables: la opinión de los administrativos es dependiente del tipo de cargo que ocupe.

Tabla 11. Relaciones coordinadas/efectivas

entre los integrantes de las diferentes áreas/departamentos

\begin{tabular}{|c|l|c|c|c|}
\cline { 2 - 5 } \multicolumn{2}{c|}{} & Frecuencia & $\%$ & \% acumulado \\
\hline \multirow{4}{*}{ Opciones de respuesta } & Nunca & 4 & 1,3 & 1,3 \\
\cline { 2 - 5 } & Rara vez & 50 & 16,8 & 18,2 \\
\cline { 2 - 5 } & Usualmente & 178 & 59,9 & 100,0 \\
\cline { 2 - 5 } & Siempre & 65 & 21,9 & 40,1 \\
\cline { 2 - 5 } & Total & 297 & 100,0 & \\
\hline
\end{tabular}

Fuente: elaboración propia 
Tabla 12. Relaciones coordinadas/efectivas

entre los integrantes de las diferentes áreas/departamentos según cargo ocupado

\begin{tabular}{|c|c|c|c|c|c|c|}
\hline & \multirow{3}{*}{$\begin{array}{c}\text { Nunca } \\
0\end{array}$} & \multirow{3}{*}{$\frac{\text { Rara vez }}{7}$} & \multirow{3}{*}{$\begin{array}{c}\text { Siempre } \\
11\end{array}$} & \multirow{3}{*}{$\begin{array}{c}\text { Usualmente } \\
16\end{array}$} & \multirow{3}{*}{$\frac{\text { Total }}{34}$} \\
\hline & & & & & & \\
\hline \multirow{5}{*}{ Cargo } & Auxiliar & & & & & \\
\hline & Jefe & 0 & 2 & 8 & 13 & 23 \\
\hline & Profesional & 3 & 25 & 16 & 71 & 115 \\
\hline & Secretario & 1 & 4 & 9 & 26 & 40 \\
\hline & Técnico & 0 & 12 & 21 & 52 & 85 \\
\hline \multicolumn{2}{|l|}{ Total } & 4 & 50 & 65 & 178 & 297 \\
\hline \multicolumn{3}{|c|}{ Prueba de independencia } & Valor & $\mathrm{Gl}$ & \multicolumn{2}{|c|}{ Sig. asintótica (bilateral) } \\
\hline \multicolumn{3}{|c|}{ Chi-cuadrado de Pearson } & $16,329^{a}$ & 12 & \multicolumn{2}{|c|}{0,177} \\
\hline \multicolumn{3}{|c|}{ Razón de verosimilitudes } & 18,187 & 12 & \multicolumn{2}{|c|}{0,110} \\
\hline \multicolumn{3}{|c|}{ N. ${ }^{\circ}$ de casos válidos } & 297 & & & \\
\hline
\end{tabular}

Fuente: elaboración propia

Pvalor $=0,177>0,05$. Se acepta la hipótesis de independencia entre las variables: la opinión de los administrativos es independiente del tipo de cargo que este ocupe.

Tabla 13. Predomina el conflicto al momento de interactuar con el jefe

\begin{tabular}{|c|l|c|c|c|}
\cline { 3 - 5 } \multicolumn{2}{c|}{} & Frecuencia & $\%$ & \% acumulado \\
\hline \multirow{4}{*}{ Opciones de respuesta } & Nunca & 132 & 44,4 & 44,4 \\
\cline { 2 - 5 } & Rara vez & 122 & 41,1 & 85,5 \\
\cline { 2 - 5 } & Usualmente & 30 & 10,1 & 100,0 \\
\cline { 2 - 5 } & Siempre & 13 & 4,4 & 89,9 \\
\cline { 2 - 5 } & Total & 297 & 100,0 & \\
\hline
\end{tabular}

Fuente: elaboración propia

\begin{tabular}{|c|l|c|c|c|c|c|}
\cline { 3 - 7 } \multicolumn{2}{c|}{} & Nunca & Rara vez & Siempre & Usualmente & Total \\
\hline \multirow{4}{*}{ Cargo } & Auxiliar & 18 & 10 & 2 & 4 & 34 \\
\cline { 2 - 7 } & Jefe & 13 & 6 & 3 & 1 & 23 \\
\cline { 2 - 7 } & Profesional & 47 & 52 & 3 & 13 & 115 \\
\cline { 2 - 7 } & Secretario & 20 & 15 & 0 & 5 & 40 \\
\cline { 2 - 7 } & Técnico & 34 & 39 & 5 & 7 & 85 \\
\hline \multirow{7}{*}{ Total } & 132 & 122 & 13 & 30 & 297 \\
\hline
\end{tabular}


Felicidad organizacional: mediación entre la valoración del empleado y la productividad. Una mirada ...

\begin{tabular}{|l|c|c|c|}
\hline \multicolumn{1}{|c|}{ Prueba de independencia } & Valor & Gl & Sig. asintótica (bilateral) \\
\hline Chi-cuadrado de Pearson & $14,428^{\mathrm{a}}$ & 12 & 0,274 \\
\hline Razón de verosimilitudes & 15,281 & 12 & 0,226 \\
\hline N. $^{\circ}$ de casos válidos & 297 & & \\
\hline
\end{tabular}

Fuente: elaboración propia

Pvalor $=0.274>0.05$. Se acepta la hipótesis de independencia entre las variables: la opinión de los administrativos es independiente del tipo de cargo que este ocupe.

Tabla 15. Predomina el conflicto al momento de interactuar con los compañeros

\begin{tabular}{|c|l|c|c|c|}
\cline { 3 - 5 } \multicolumn{2}{c|}{} & Frecuencia & $\%$ & \% acumulado \\
\hline \multirow{4}{*}{ Opciones de respuesta } & Nunca & 134 & 45,1 & 45,1 \\
\cline { 2 - 5 } & Rara vez & 130 & 43,8 & 88,9 \\
\cline { 2 - 5 } & Usualmente & 23 & 7,7 & 100,0 \\
\cline { 2 - 5 } & Siempre & 10 & 3,4 & 92,3 \\
\cline { 2 - 5 } & Total & 297 & 100,0 & \\
\hline
\end{tabular}

Fuente: elaboración propia

Tabla 16. Predomina el conflicto al momento de interactuar con los compañeros

\begin{tabular}{|c|l|c|c|c|c|c|}
\cline { 3 - 7 } \multicolumn{2}{c|}{} & Nunca & Rara vez & Siempre & Usualmente & Total \\
\hline \multirow{4}{*}{ Cargo } & Auxiliar & 13 & 14 & 2 & 5 & 34 \\
\cline { 2 - 7 } & Jefe & 15 & 4 & 3 & 1 & 23 \\
\cline { 2 - 7 } & Profesional & 51 & 55 & 1 & 8 & 115 \\
\cline { 2 - 7 } & Secretario & 20 & 18 & 0 & 2 & 40 \\
\cline { 2 - 7 } & Técnico & 35 & 39 & 4 & 7 & 85 \\
\hline \multirow{5}{*}{ Total } & 134 & 130 & 10 & 23 & 297 \\
\hline
\end{tabular}

\begin{tabular}{|l|c|c|c|}
\hline \multicolumn{1}{|c|}{ Prueba de independencia } & Valor & Gl & Sig. asintótica (bilateral) \\
\hline Chi-cuadrado de Pearson & $21,120^{\text {a }}$ & 12 & 0,049 \\
\hline Razón de verosimilitudes & 21,009 & 12 & 0,050 \\
\hline N. $^{\circ}$ de casos válidos & 297 & & \\
\hline
\end{tabular}

Fuente: elaboración propia

Pvalor $=0,049<0,05$. Se rechaza la hipótesis de independencia entre las variables: la opinión de los administrativos es dependiente del tipo de cargo que este ocupe. 


\section{Variable 2. Satisfacción laboral}

Tabla 17. Desarrollo de acciones que propician el bienestar laboral

\begin{tabular}{|c|l|c|c|c|}
\cline { 3 - 5 } \multicolumn{2}{c|}{} & Frecuencia & $\%$ & \% acumulado \\
\hline \multirow{4}{*}{ Opciones de respuesta } & Nunca & 6 & 2,0 & 2,0 \\
\cline { 2 - 5 } & Rara vez & 63 & 21,2 & 23,2 \\
\cline { 2 - 5 } & Usualmente & 169 & 56,9 & 100,0 \\
\cline { 2 - 5 } & Siempre & 59 & 19,9 & 43,1 \\
\cline { 2 - 5 } & Total & 297 & 100,0 & \\
\hline
\end{tabular}

Fuente: elaboración propia

Tabla 18. Desarrollo de acciones que propician el bienestar laboral según cargo ocupado

\begin{tabular}{|c|l|c|c|c|c|c|}
\cline { 2 - 7 } \multicolumn{2}{c|}{} & Nunca & Rara vez & Siempre & Usualmente & Total \\
\hline \multirow{4}{*}{ Cargo } & Auxiliar & 2 & 6 & 8 & 18 & 34 \\
\cline { 2 - 7 } & Jefe & 0 & 2 & 7 & 14 & 23 \\
\cline { 2 - 7 } & Profesional & 2 & 32 & 22 & 59 & 115 \\
\cline { 2 - 7 } & Secretario & 0 & 7 & 4 & 29 & 40 \\
\cline { 2 - 7 } & Técnico & 2 & 16 & 18 & 49 & 85 \\
\hline \multirow{7}{*}{ Total } & 6 & 63 & 59 & 169 & 297 \\
\hline
\end{tabular}

\begin{tabular}{|l|c|c|c|}
\hline \multicolumn{1}{|c|}{ Prueba de independencia } & Valor & $\mathrm{Gl}$ & Sig. asintótica (bilateral) \\
\hline Chi-cuadrado de Pearson & $14,734^{\mathrm{a}}$ & 12 & 0,256 \\
\hline Razón de verosimilitudes & 15,590 & 12 & 0,211 \\
\hline N. $^{\circ}$ de casos válidos & 297 & & \\
\hline
\end{tabular}

Fuente: elaboración propia

Pvalor $=0,256>0,05$. Se acepta la hipótesis de independencia entre las variables: la opinión de los administrativos es independiente del tipo de cargo que este ocupe.

Tabla 19. Existencia de reconocimientos, méritos o estímulos por el buen desempeño laboral

\begin{tabular}{|c|l|c|c|c|}
\cline { 2 - 5 } \multicolumn{2}{c|}{} & Frecuencia & $\%$ & \% acumulado \\
\hline \multirow{4}{*}{ Opciones de respuesta } & Nunca & 48 & 16,2 & 16,2 \\
\cline { 2 - 5 } & Rara vez & 95 & 32,0 & 48,1 \\
\cline { 2 - 5 } & Usualmente & 108 & 36,4 & 100,0 \\
\cline { 2 - 5 } & Siempre & 46 & 15,5 & 63,6 \\
\cline { 2 - 5 } & Total & 297 & 100,0 & \\
\hline
\end{tabular}

Fuente: elaboración propia 
Felicidad organizacional: mediación entre la valoración del empleado y la productividad. Una mirada ...

Tabla 20. Existencia de reconocimientos, méritos o estímulos por el buen desempeño laboral según cargo ocupado

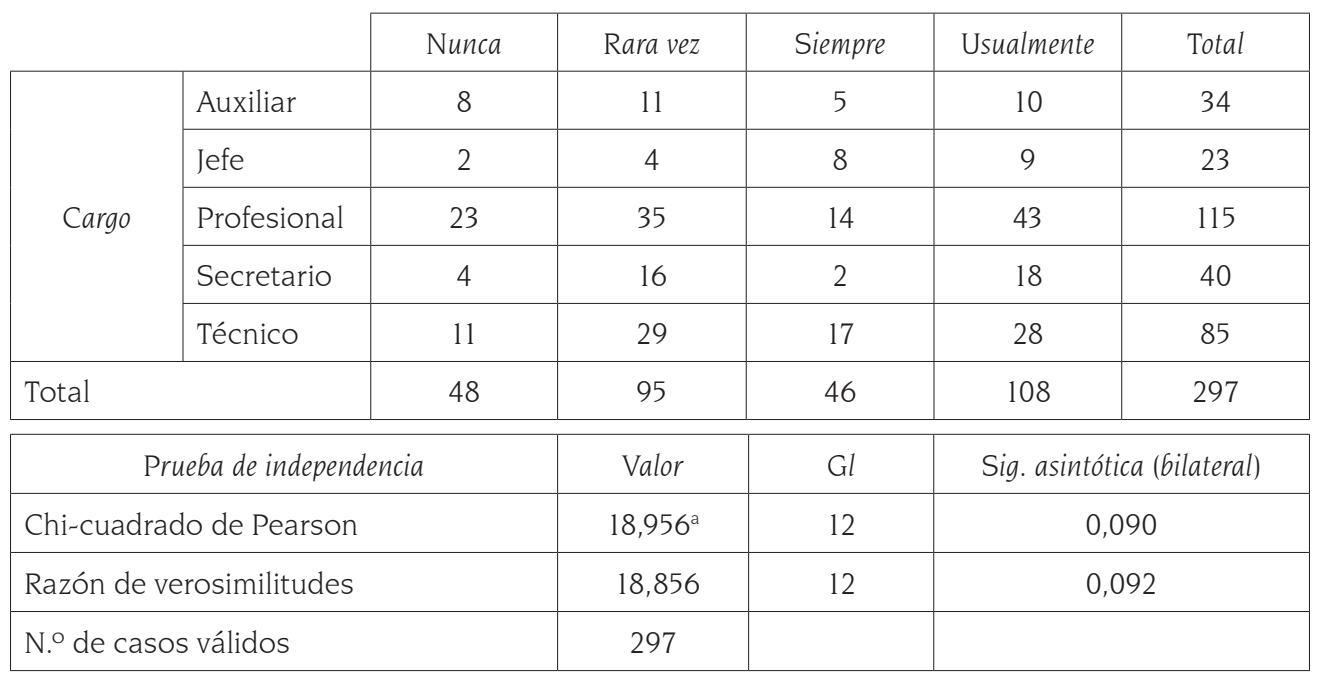

Fuente: elaboración propia

Pvalor $=0.090>0.05$. Se acepta la hipótesis de independencia entre las variables: la opinión de los administrativos es independiente del tipo de cargo que este ocupe.

Tabla 21. La felicidad como valor que se promueve en la organización

\begin{tabular}{|c|l|c|c|c|}
\cline { 3 - 5 } \multicolumn{2}{c|}{} & Frecuencia & $\%$ & \% acumulado \\
\hline \multirow{4}{*}{ Opciones de respuesta } & Nunca & 48 & 16,2 & 16,2 \\
\cline { 2 - 5 } & Rara vez & 108 & 36,4 & 52,5 \\
\cline { 2 - 5 } & Usualmente & 103 & 34,7 & 100,0 \\
\cline { 2 - 5 } & Siempre & 38 & 12,8 & 65,3 \\
\cline { 2 - 5 } & Total & 297 & 100,0 & \\
\hline
\end{tabular}

Fuente: elaboración propia

Tabla 22. La felicidad como valor que se promueve en la organización según cargo ocupado

\begin{tabular}{|c|l|c|c|c|c|c|}
\cline { 3 - 7 } \multicolumn{2}{c|}{} & Nunca & Rara vez & Siempre & Usualmente & Total \\
\hline \multirow{4}{*}{ Cargo } & Auxiliar & 13 & 8 & 3 & 10 & 34 \\
\cline { 2 - 7 } & Jefe & 1 & 5 & 6 & 11 & 23 \\
\cline { 2 - 7 } & Profesional & 22 & 46 & 10 & 37 & 115 \\
\cline { 2 - 7 } & Secretario & 5 & 16 & 6 & 13 & 40 \\
\cline { 2 - 7 } & Técnico & 7 & 33 & 13 & 32 & 85 \\
\hline \multirow{7}{*}{ Total } & 48 & 108 & 38 & 103 & 297 \\
\hline
\end{tabular}




\begin{tabular}{|l|c|c|c|}
\hline \multicolumn{1}{|c|}{ Prueba de independencia } & Valor & Gl & Sig. asintótica (bilateral) \\
\hline Chi-cuadrado de Pearson & $27,667^{\text {a }}$ & 12 & 0,006 \\
\hline Razón de verosimilitudes & 26,138 & 12 & 0,010 \\
\hline N. ${ }^{\circ}$ de casos válidos & 297 & & \\
\hline
\end{tabular}

Fuente: elaboración propia

Pvalor $=0,006<0,05$. Se rechaza la hipótesis de independencia entre las variables: la opinión de los administrativos es dependiente del tipo de cargo que este ocupe.

Tabla 23. La universidad contribuye a su desarrollo personal, familiar y profesional aportándole un salario emocional, comprendido este como el conjunto de retribuciones no económicas que el trabajador puede obtener de la empresa, cuyo objetivo es incentivar de forma positiva la imagen que tiene sobre su ambiente laboral, como, por ejemplo: horarios flexibles, teletrabajo, capacitaciones no asociadas directamente al trabajo y reconocimiento al trabajo bien hecho.

\begin{tabular}{|c|l|c|c|c|}
\cline { 3 - 5 } \multicolumn{2}{c|}{} & Frecuencia & $\%$ & \% acumulado \\
\hline \multirow{4}{*}{ Opciones de respuesta } & Nunca & 28 & 9,4 & 9,4 \\
\cline { 2 - 5 } & Rara vez & 141 & 47,5 & 56,9 \\
\cline { 2 - 5 } & Usualmente & 91 & 30,6 & 100,0 \\
\cline { 2 - 5 } & Siempre & 37 & 12,5 & 69,4 \\
\cline { 2 - 5 } & Total & 297 & 100,0 & \\
\hline
\end{tabular}

Fuente: elaboración propia

Tabla 24. La empresa contribuye a su desarrollo personal, familiar y profesional aportándole un salario emocional según cargo ocupado

\begin{tabular}{|c|l|c|c|c|c|c|}
\cline { 2 - 7 } \multicolumn{2}{c|}{} & Nunca & Rara vez & Siempre & Usualmente & Total \\
\hline \multirow{4}{*}{ Cargo } & Auxiliar & 6 & 17 & 5 & 6 & 34 \\
\cline { 2 - 7 } & Jefe & 0 & 9 & 7 & 7 & 23 \\
\cline { 2 - 7 } & Profesional & 19 & 47 & 12 & 37 & 115 \\
\cline { 2 - 7 } & Secretario & 3 & 22 & 2 & 13 & 40 \\
\cline { 2 - 7 } & Técnico & 0 & 46 & 11 & 28 & 85 \\
\hline \multirow{7}{*}{ Total } & 28 & 141 & 37 & 91 & 297 \\
\hline
\end{tabular}

\begin{tabular}{|l|c|c|c|}
\hline \multicolumn{1}{|c|}{ Prueba de independencia } & Valor & Gl & Sig. asintótica (bilateral) \\
\hline Chi-cuadrado de Pearson & $32,060^{\text {a }}$ & 12 & 0,001 \\
\hline Razón de verosimilitudes & 39,779 & 12 & 0,000 \\
\hline N. $^{\circ}$ de casos válidos & 297 & & \\
\hline
\end{tabular}

Fuente: elaboración propia 
Felicidad organizacional: mediación entre la valoración del empleado y la productividad. Una mirada ...

Pvalor $=0,001<0,05$. Se rechaza la hipótesis de independencia entre las variables: la opinión de los administrativos es dependiente del tipo de cargo que este ocupe.

Tabla 25. Promoción para el ascenso ligado al servilismo

\begin{tabular}{|c|l|c|c|c|}
\cline { 3 - 5 } \multicolumn{2}{c|}{} & Frecuencia & $\%$ & \% acumulado \\
\hline \multirow{4}{*}{ Opciones de respuesta } & Nunca & 30 & 10,1 & 10,1 \\
\cline { 2 - 5 } & Rara vez & 77 & 25,9 & 36,0 \\
\cline { 2 - 5 } & Usualmente & 106 & 35,7 & 100,0 \\
\cline { 2 - 5 } & Siempre & 84 & 28,3 & 64,3 \\
\cline { 2 - 5 } & Total & 297 & 100,0 & \\
\hline
\end{tabular}

Fuente: elaboración propia

Tabla 26. Promoción para el ascenso ligado al servilismo según cargo ocupado

\begin{tabular}{|c|l|c|c|c|c|c|}
\cline { 3 - 7 } \multicolumn{2}{c|}{} & Nunca & Rara vez & Siempre & Usualmente & Total \\
\hline \multirow{5}{*}{ Cargo } & Auxiliar & 5 & 5 & 12 & 12 & 34 \\
\cline { 2 - 7 } & Jefe & 6 & 7 & 7 & 3 & 23 \\
\cline { 2 - 7 } & Profesional & 9 & 27 & 33 & 46 & 115 \\
\cline { 2 - 7 } & Secretario & 1 & 13 & 10 & 16 & 40 \\
\cline { 2 - 7 } & Técnico & 9 & 25 & 22 & 29 & 85 \\
\hline \multirow{7}{*}{ Total } & 30 & 77 & 84 & 106 & 297 \\
\hline
\end{tabular}

\begin{tabular}{|l|c|c|c|}
\hline \multicolumn{1}{|c|}{ Prueba de independencia } & Valor & Gl & Sig. asintótica (bilateral) \\
\hline Chi-cuadrado de Pearson & $17,725^{\mathrm{a}}$ & 12 & 0,124 \\
\hline Razón de verosimilitudes & 18,150 & 12 & 0,111 \\
\hline N. $^{\circ}$ de casos válidos & 297 & & \\
\hline
\end{tabular}

Fuente: elaboración propia

Pvalor $=0,124>0,05$. Se acepta la hipótesis de independencia entre las variables: la opinión de los administrativos es independiente del tipo de cargo que este ocupe.

Tabla 27. Recibe de manera justa su salario

\begin{tabular}{|c|l|c|c|c|}
\cline { 3 - 5 } \multicolumn{2}{c|}{} & Frecuencia & $\%$ & \% acumulado \\
\hline \multirow{4}{*}{ Opciones de respuesta } & Nunca & 29 & 9,8 & 9,8 \\
\cline { 2 - 5 } & Rara vez & 101 & 34,0 & 43,8 \\
\cline { 2 - 5 } & Usualmente & 110 & 37,0 & 100,0 \\
\cline { 2 - 5 } & Siempre & 57 & 19,2 & 63,0 \\
\cline { 2 - 5 } & Total & 297 & 100,0 & \\
\hline
\end{tabular}

Fuente: elaboración propia 
Tabla 28. Salario justo según cargo ocupado

\begin{tabular}{|c|l|c|c|c|c|c|}
\cline { 3 - 7 } \multicolumn{2}{c|}{} & Nunca & Rara vez & Siempre & Usualmente & Total \\
\hline \multirow{5}{*}{ Cargo } & Auxiliar & 4 & 12 & 7 & 11 & 34 \\
\cline { 2 - 7 } & Jefe & 0 & 1 & 12 & 10 & 23 \\
\cline { 2 - 7 } & Profesional & 14 & 38 & 15 & 48 & 115 \\
\cline { 2 - 7 } & Secretario & 6 & 13 & 5 & 16 & 40 \\
\cline { 2 - 7 } & Técnico & 5 & 37 & 18 & 25 & 85 \\
\hline \multirow{7}{*}{ Total } & 29 & 101 & 57 & 110 & 297 \\
\hline
\end{tabular}

\begin{tabular}{|l|c|c|c|}
\hline \multicolumn{1}{|c|}{ Prueba de independencia } & Valor & Gl & Sig. asintótica (bilateral) \\
\hline Chi-cuadrado de Pearson & $32,813^{\text {a }}$ & 12 & 0,001 \\
\hline Razón de verosimilitudes & 34,733 & 12 & 0,001 \\
\hline N. $^{\circ}$ de casos válidos & 297 & & \\
\hline
\end{tabular}

Fuente: elaboración propia

Pvalor $=0,001<0,05$. Se rechaza la hipótesis de independencia entre las variables: la opinión de los administrativos es dependiente del tipo de cargo que este ocupe.

Tabla 29. Remuneración basada más en relaciones personales que en los méritos y resultados

\begin{tabular}{|c|l|c|c|c|}
\cline { 3 - 5 } \multicolumn{2}{c|}{} & Frecuencia & $\%$ & \% acumulado \\
\hline \multirow{4}{*}{ Opciones de respuesta } & Nunca & 43 & 14,5 & 14,5 \\
\cline { 2 - 5 } & Rara vez & 66 & 22,2 & 36,7 \\
\cline { 2 - 5 } & Usualmente & 78 & 26,3 & 100,0 \\
\cline { 2 - 5 } & Siempre & 110 & 37,0 & 73,7 \\
\cline { 2 - 5 } & Total & 297 & 100,0 & \\
\hline
\end{tabular}

Fuente: elaboración propia

Tabla 30. Remuneración basada más en relaciones personales que en los méritos y resultados según cargo ocupado

\begin{tabular}{|c|l|c|c|c|c|c|}
\cline { 3 - 7 } \multicolumn{2}{c|}{} & Nunca & Rara vez & Siempre & Usualmente & Total \\
\hline \multirow{4}{*}{ Cargo } & Auxiliar & 8 & 8 & 14 & 4 & 34 \\
\cline { 2 - 7 } & Jefe & 8 & 4 & 6 & 5 & 23 \\
\cline { 2 - 7 } & Profesional & 16 & 21 & 45 & 33 & 115 \\
\cline { 2 - 7 } & Secretario & 3 & 15 & 15 & 7 & 40 \\
\cline { 2 - 7 } & Técnico & 8 & 18 & 30 & 29 & 85 \\
\hline \multirow{3}{*}{ Total } & 43 & 66 & 110 & 78 & 297 \\
\hline
\end{tabular}


Felicidad organizacional: mediación entre la valoración del empleado y la productividad. Una mirada ...

\begin{tabular}{|l|c|c|c|}
\hline \multicolumn{1}{|c|}{ Prueba de independencia } & Valor & Gl & Sig. asintótica (bilateral) \\
\hline Chi-cuadrado de Pearson & $24,110^{\text {a }}$ & 12 & 0,020 \\
\hline Razón de verosimilitudes & 22,568 & 12 & 0,032 \\
\hline N. $^{\circ}$ de casos válidos & 297 & & \\
\hline
\end{tabular}

Fuente: elaboración propia

Pvalor $=0,020<0,05$. Se rechaza la hipótesis de independencia entre las variables: la opinión de los administrativos es dependiente del tipo de cargo que este ocupe.

\section{Variable 3. Compromiso con la función y la organización}

Tabla 31. Aporte del desempeño personal al desarrollo de la organización

\begin{tabular}{|c|l|c|c|c|}
\cline { 3 - 5 } \multicolumn{2}{c|}{} & Frecuencia & $\%$ & \% acumulado \\
\hline \multirow{4}{*}{ Opciones de respuesta } & Nunca & & & \\
\cline { 2 - 5 } & Rara vez & 5 & 1,7 & 1,7 \\
\cline { 2 - 5 } & Usualmente & 88 & 29,6 & 100,0 \\
\cline { 2 - 5 } & Siempre & 204 & 68,7 & 70,4 \\
\cline { 2 - 5 } & Total & 297 & 100,0 & \\
\hline
\end{tabular}

Fuente: elaboración propia

Tabla 32. Aporte del desempeño personal al desarrollo de la organización según cargo ocupado

\begin{tabular}{|c|c|c|c|c|c|c|}
\hline & \multirow{3}{*}{ Nunca } & \multirow{3}{*}{$\begin{array}{c}\text { Rara vez } \\
1\end{array}$} & \multirow{3}{*}{$\begin{array}{c}\text { Siempre } \\
19\end{array}$} & \multirow{3}{*}{$\begin{array}{c}\text { Usualmente } \\
14\end{array}$} & \multirow{3}{*}{$\frac{\text { Total }}{34}$} \\
\hline & & & & & & \\
\hline \multirow{5}{*}{ Cargo } & Auxiliar & & & & & \\
\hline & Jefe & & 0 & 20 & 3 & 23 \\
\hline & Profesional & & 3 & 90 & 22 & 115 \\
\hline & Secretario & & 0 & 26 & 14 & 40 \\
\hline & Técnico & & 1 & 49 & 35 & 85 \\
\hline \multicolumn{2}{|l|}{ Total } & & 5 & 204 & 88 & 297 \\
\hline \multicolumn{3}{|c|}{ Prueba de independencia } & Valor & $\mathrm{Gl}$ & \multicolumn{2}{|c|}{ Sig. asintótica (bilateral) } \\
\hline \multicolumn{3}{|c|}{ Chi-cuadrado de Pearson } & $19,304^{\mathrm{a}}$ & 8 & \multicolumn{2}{|c|}{0,013} \\
\hline \multicolumn{3}{|c|}{ Razón de verosimilitudes } & 20,811 & 8 & \multicolumn{2}{|c|}{0,008} \\
\hline \multicolumn{3}{|c|}{ N. ${ }^{\circ}$ de casos válidos } & 297 & & & \\
\hline
\end{tabular}

Fuente: elaboración propia

Pvalor $=0,013<0,05$. Se rechaza la hipótesis de independencia entre las variables: la opinión de los administrativos es dependiente del tipo de cargo que este ocupe. 
Tabla 33. Uso de capacidades y competencias para el desarrollo del trabajo

\begin{tabular}{|c|l|c|c|c|}
\cline { 3 - 5 } \multicolumn{2}{c|}{} & Frecuencia & $\%$ & \% acumulado \\
\hline \multirow{3}{*}{ Opciones de respuesta } & Nunca & 2 & 0,7 & 0,7 \\
\cline { 2 - 5 } & Rara vez & 11 & 3,7 & 4,4 \\
\cline { 2 - 5 } & Usualmente & 82 & 27,6 & 100,0 \\
\cline { 2 - 5 } & Siempre & 202 & 68,0 & 72,4 \\
\cline { 2 - 5 } & Total & 297 & 100,0 & \\
\hline
\end{tabular}

Fuente: elaboración propia

Tabla 34. Uso de capacidades y competencias para el desarrollo del trabajo según cargo ocupado

\begin{tabular}{|c|l|c|c|c|c|c|}
\cline { 3 - 7 } \multicolumn{2}{c|}{} & Nunca & Rara vez & Siempre & Usualmente & Total \\
\hline \multirow{4}{*}{ Cargo } & Auxiliar & 0 & 3 & 18 & 13 & 34 \\
\cline { 2 - 7 } & Jefe & 0 & 0 & 19 & 4 & 23 \\
\cline { 2 - 7 } & Profesional & 1 & 2 & 88 & 24 & 115 \\
\cline { 2 - 7 } & Secretario & 1 & 2 & 25 & 12 & 40 \\
\cline { 2 - 7 } & Técnico & 0 & 4 & 52 & 29 & 85 \\
\hline \multirow{7}{*}{ Total } & 2 & 11 & 202 & 82 & 297 \\
\hline
\end{tabular}

\begin{tabular}{|l|c|c|c|}
\hline \multicolumn{1}{|c|}{ Prueba de independencia } & Valor & Gl & Sig. asintótica (bilateral) \\
\hline Chi-cuadrado de Pearson & $17,254^{\text {a }}$ & 12 & 0,140 \\
\hline Razón de verosimilitudes & 17,908 & 12 & 0,119 \\
\hline N. ${ }^{\circ}$ de casos válidos & 297 & & \\
\hline
\end{tabular}

Fuente: elaboración propia

Pvalor $=0.140>0.05$. Se acepta la hipótesis de independencia entre las variables: la opinión de los administrativos es independiente del tipo de cargo que este ocupe.

Tabla 35. Apropiación de los problemas que surgen en la organización

\begin{tabular}{|c|l|c|c|c|}
\cline { 3 - 5 } \multicolumn{2}{c|}{} & Frecuencia & $\%$ & \% acumulado \\
\hline \multirow{3}{*}{ Opciones de respuesta } & Nunca & 3 & 1,0 & 1,0 \\
\cline { 2 - 5 } & Rara vez & 28 & 9,4 & 10,4 \\
\cline { 2 - 5 } & Usualmente & 128 & 43,1 & 100,0 \\
\cline { 2 - 5 } & Siempre & 138 & 46,5 & 56,9 \\
\cline { 2 - 5 } & Total & 297 & 100,0 & \\
\hline
\end{tabular}

Fuente: elaboración propia, 2017 
Felicidad organizacional: mediación entre la valoración del empleado y la productividad. Una mirada ...

Tabla 36. Apropiación de los problemas que surgen en la organización según cargo ocupado

\begin{tabular}{|c|c|c|c|c|c|c|}
\hline & \multirow{3}{*}{$\begin{array}{c}\text { Nunca } \\
1\end{array}$} & \multirow{3}{*}{$\frac{\text { Rara vez }}{4}$} & \multirow{3}{*}{$\begin{array}{c}\text { Siempre } \\
14\end{array}$} & \multirow{3}{*}{$\begin{array}{c}\text { Usualmente } \\
15\end{array}$} & \multirow{3}{*}{$\begin{array}{c}\text { Total } \\
34\end{array}$} \\
\hline & & & & & & \\
\hline \multirow{5}{*}{ Cargo } & Auxiliar & & & & & \\
\hline & Jefe & 0 & 1 & 18 & 4 & 23 \\
\hline & Profesional & 1 & 8 & 52 & 54 & 115 \\
\hline & Secretario & 0 & 5 & 19 & 16 & 40 \\
\hline & Técnico & 1 & 10 & 35 & 39 & 85 \\
\hline \multicolumn{2}{|l|}{ Total } & 3 & 28 & 138 & 128 & 297 \\
\hline \multicolumn{3}{|c|}{ Prueba de independencia } & Valor & $\mathrm{Gl}$ & \multicolumn{2}{|c|}{ Sig. asintótica (bilateral) } \\
\hline \multicolumn{3}{|c|}{ Chi-cuadrado de Pearson } & $14,344^{\mathrm{a}}$ & 12 & \multicolumn{2}{|c|}{279} \\
\hline \multicolumn{3}{|c|}{ Razón de verosimilitudes } & 14,889 & 12 & \multicolumn{2}{|c|}{,248 } \\
\hline \multicolumn{3}{|c|}{ N. ${ }^{\circ}$ de casos válidos } & 297 & & & \\
\hline
\end{tabular}

Fuente: elaboración propia

Pvalor $=0,279>0,05$. Se acepta la hipótesis de independencia entre las variables: la opinión de los administrativos es independiente del tipo de cargo que este ocupe.

Tabla 37. Aporte del desempeño personal al desarrollo de la organización

\begin{tabular}{|c|l|c|c|c|}
\cline { 3 - 5 } \multicolumn{2}{c|}{} & Frecuencia & $\%$ & \% acumulado \\
\hline \multirow{3}{*}{ Opciones de respuesta } & Nunca & 4 & 1,3 & 1,3 \\
\cline { 2 - 5 } & Rara vez & 34 & 11,4 & 12,8 \\
\cline { 2 - 5 } & Usualmente & 127 & 42,8 & 100,0 \\
\cline { 2 - 5 } & Siempre & 132 & 44,4 & 57,2 \\
\cline { 2 - 5 } & Total & 297 & 100,0 & \\
\hline
\end{tabular}

Fuente: elaboración propia, 2017.

Tabla 38. Aporte del desempeño personal al desarrollo de la organización según cargo ocupado

\begin{tabular}{|c|c|c|c|c|c|c|}
\hline & & Nunca & Rara vez & Siempre & Usualmente & Total \\
\hline \multirow{5}{*}{ Cargo } & Auxiliar & & 1 & 19 & 14 & 34 \\
\hline & Jefe & & 0 & 20 & 3 & 23 \\
\hline & Profesional & & 3 & 90 & 22 & 115 \\
\hline & Secretario & & 0 & 26 & 14 & 40 \\
\hline & Técnico & & 1 & 49 & 35 & 85 \\
\hline
\end{tabular}




\begin{tabular}{|l|c|c|c|c|}
\hline Total & 5 & 204 & 88 & 297 \\
\hline \multicolumn{1}{|c|}{ Prueba de independencia } & Valor & Gl & Sig. asintótica (6ilateral) \\
\hline Chi-cuadrado de Pearson & $19,304^{\mathrm{a}}$ & 8 & 0,013 \\
\hline Razón de verosimilitudes & 20,811 & 8 & 0,008 \\
\hline N. ${ }^{\circ}$ de casos válidos & 297 & & \\
\hline
\end{tabular}

Fuente: elaboración propia

Pvalor $=0,013<0,05$. Se rechaza la hipótesis de independencia entre las variables: la opinión de los administrativos es dependiente del tipo de cargo que este ocupe.

Tabla 39. Interés personal en los resultados obtenidos por la organización

\begin{tabular}{|c|l|c|c|c|}
\cline { 3 - 5 } \multicolumn{2}{c|}{} & Frecuencia & $\%$ & \% acumulado \\
\hline \multirow{4}{*}{ Opciones de respuesta } & Nunca & & & \\
\cline { 2 - 5 } & Rara vez & 22 & 7,4 & 7,4 \\
\cline { 2 - 5 } & Usualmente & 102 & 34,3 & 100,0 \\
\cline { 2 - 5 } & Siempre & 173 & 58,2 & 65,7 \\
\cline { 2 - 5 } & Total & 297 & 100,0 & \\
\hline
\end{tabular}

Fuente: elaboración propia

Tabla 40. Interés personal en los resultados obtenidos por la organización según cargo ocupado

\begin{tabular}{|l|l|c|c|c|c|c|}
\cline { 3 - 7 } \multicolumn{2}{c|}{} & Nunca & Rara vez & Siempre & Usualmente & Total \\
\hline \multirow{4}{*}{} & Auxiliar & & 3 & 18 & 13 & 34 \\
\cline { 2 - 7 } Cargo & Jefe & & 1 & 18 & 4 & 23 \\
\cline { 2 - 7 } & Profesional & & 7 & 66 & 42 & 115 \\
\cline { 2 - 7 } & Secretario & & 4 & 23 & 13 & 40 \\
\cline { 2 - 7 } & Técnico & & 7 & 48 & 30 & 85 \\
\hline Total & & 22 & 173 & 102 & 297 \\
\hline
\end{tabular}

\begin{tabular}{|l|c|c|c|}
\hline \multicolumn{1}{|c|}{ Prueba de independencia } & Valor & Gl & Sig. asintótica (bilateral) \\
\hline Chi-cuadrado de Pearson & $5,201^{\text {a }}$ & 8 & 0,736 \\
\hline Razón de verosimilitudes & 5,498 & 8 & 0,703 \\
\hline N. $^{\circ}$ de casos válidos & 297 & & \\
\hline
\end{tabular}

Fuente: elaboración propia

Pvalor $=0,736>0,05$. Se acepta la hipótesis de independencia entre las varia- 
bles: la opinión de los administrativos es independiente del tipo de cargo que este ocupe.

\section{Variable 4. Innovación}

Tabla 41. Probar nuevos métodos para el desarrollo de su trabajo

\begin{tabular}{|c|l|c|c|c|}
\cline { 3 - 5 } \multicolumn{2}{c|}{} & Frecuencia & $\%$ & \% acumulado \\
\hline \multirow{4}{*}{ Opciones de respuesta } & Nunca & 3 & 1,0 & 1,0 \\
\cline { 2 - 5 } & Rara vez & 51 & 17,2 & 18,2 \\
\cline { 2 - 5 } & Usualmente & 157 & 52,9 & 100,0 \\
\cline { 2 - 5 } & Siempre & 86 & 29,0 & 47,1 \\
\cline { 2 - 5 } & Total & 297 & 100,0 & \\
\hline
\end{tabular}

Fuente: elaboración propia

Tabla 42. Probar nuevos métodos para el desarrollo de su trabajo según cargo ocupado

\begin{tabular}{|c|l|c|c|c|c|c|}
\cline { 3 - 7 } \multicolumn{2}{c|}{} & Nunca & Rara vez & Siempre & Usualmente & Total \\
\hline \multirow{4}{*}{ Cargo } & Auxiliar & 0 & 8 & 11 & 15 & 34 \\
\cline { 2 - 7 } & Jefe & 0 & 1 & 11 & 11 & 23 \\
\cline { 2 - 7 } & Profesional & 4 & 13 & 42 & 56 & 115 \\
\cline { 2 - 7 } & Secretario & 1 & 9 & 13 & 17 & 40 \\
\cline { 2 - 7 } & Técnico & 2 & 14 & 23 & 46 & 85 \\
\hline \multirow{7}{*}{ Total } & 7 & 45 & 100 & 145 & 297 \\
\hline
\end{tabular}

\begin{tabular}{|l|c|c|c|}
\hline \multicolumn{1}{|c|}{ Prueba de independencia } & Valor & Gl & Sig. asintótica (bilateral) \\
\hline Chi-cuadrado de Pearson & $11,713^{\mathrm{a}}$ & 12 & 0,469 \\
\hline Razón de verosimilitudes & 13,285 & 12 & 0,349 \\
\hline N. ${ }^{\circ}$ de casos válidos & 297 & & \\
\hline
\end{tabular}

Fuente: elaboración propia

Pvalor $=0,469>0,05$. Se acepta la hipótesis de independencia entre las variables: la opinión de los administrativos es independiente del tipo de cargo que este ocupe.

Tabla 43. La organización fomenta acciones para promover la innovación

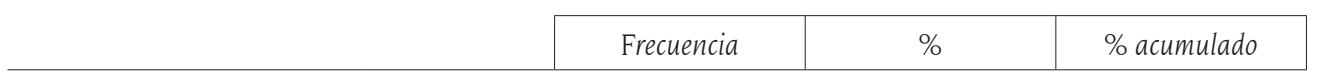




\begin{tabular}{|c|l|c|c|c|}
\hline \multirow{4}{*}{ Opciones de respuesta } & Nunca & 37 & 12,5 & 12,5 \\
\cline { 2 - 5 } & Rara vez & 101 & 34,0 & 46,5 \\
\cline { 2 - 5 } & Usualmente & 115 & 38,7 & 100,0 \\
\cline { 2 - 5 } & Siempre & 44 & 14,8 & 61,3 \\
\cline { 2 - 5 } & Total & 297 & 100,0 & \\
\hline
\end{tabular}

Fuente: elaboración propia

Tabla 44. Aporte del desempeño personal al desarrollo de la organización según cargo ocupado

\begin{tabular}{|c|c|c|c|c|c|c|}
\hline & Nunca & Rara vez & Siempre & Usualmente & Total \\
\hline \multirow{5}{*}{ Cargo } & Auxiliar & 10 & 11 & 4 & 9 & 34 \\
\hline & Jefe & 0 & 4 & 9 & 10 & 23 \\
\hline & Profesional & 14 & 44 & 10 & 47 & 115 \\
\hline & Secretario & 4 & 14 & 4 & 18 & 40 \\
\hline & Técnico & 9 & 28 & 17 & 31 & 85 \\
\hline \multicolumn{2}{|l|}{ Total } & 37 & 101 & 44 & 115 & 297 \\
\hline \multicolumn{3}{|c|}{ Prueba de independencia } & Valor & $\mathrm{Gl}$ & \multicolumn{2}{|c|}{ Sig. asintótica (Eilateral) } \\
\hline \multicolumn{3}{|c|}{ Chi-cuadrado de Pearson } & $30,272^{\mathrm{a}}$ & 12 & \multicolumn{2}{|c|}{0,003} \\
\hline \multicolumn{3}{|c|}{ Razón de verosimilitudes } & 29,074 & 12 & \multicolumn{2}{|c|}{0,004} \\
\hline \multicolumn{3}{|c|}{ N. ${ }^{\circ}$ de casos válidos } & 297 & & & \\
\hline
\end{tabular}

Fuente: elaboración propia

Pvalor $=0,003<0,05$. Se rechaza la hipótesis de independencia entre las variables: la opinión de los administrativos es dependiente del tipo de cargo que este ocupe.

Tabla 45. Estímulo por parte de la organización para asumir riesgos razonables al intentar crear nuevas ideas

\begin{tabular}{|c|l|c|c|c|}
\cline { 3 - 5 } \multicolumn{2}{c|}{} & Frecuencia & $\%$ & \% acumulado \\
\hline \multirow{4}{*}{ Opciones de respuesta } & Nunca & 47 & 15,8 & 15,8 \\
\cline { 2 - 5 } & Rara vez & 110 & 37,0 & 52,9 \\
\cline { 2 - 5 } & Usualmente & 105 & 35,4 & 100,0 \\
\cline { 2 - 5 } & Siempre & 35 & 11,8 & 64,6 \\
\cline { 2 - 5 } & Total & 297 & 100,0 & \\
\hline
\end{tabular}

Fuente: elaboración propia

Tabla 46. Estímulo por parte de la organización

para asumir riesgos razonables al intentar crear nuevas ideas según cargo ocupado 
Felicidad organizacional: mediación entre la valoración del empleado y la productividad. Una mirada ...

\begin{tabular}{|c|l|c|c|c|c|c|}
\cline { 3 - 7 } \multicolumn{2}{c|}{} & Nunca & Rara vez & Siempre & Usualmente & Total \\
\hline \multirow{4}{*}{ Cargo } & Auxiliar & 8 & 11 & 4 & 11 & 34 \\
\cline { 2 - 7 } & Jefe & 2 & 3 & 7 & 11 & 23 \\
\cline { 2 - 7 } & Profesional & 22 & 44 & 8 & 41 & 115 \\
\cline { 2 - 7 } & Secretario & 9 & 16 & 5 & 10 & 40 \\
\cline { 2 - 7 } & Técnico & 6 & 36 & 11 & 32 & 85 \\
\hline \multirow{7}{*}{ Total } & 47 & 110 & 35 & 105 & 297 \\
\hline
\end{tabular}

\begin{tabular}{|l|c|c|c|}
\hline \multicolumn{1}{|c|}{ Prueba de independencia } & Valor & Gl & Sig. asintótica (bilateral) \\
\hline Chi-cuadrado de Pearson & $24,246^{\mathrm{a}}$ & 12 &, 019 \\
\hline Razón de verosimilitudes & 24,653 & 12 &, 017 \\
\hline N. ${ }^{\circ}$ de casos válidos & 297 & & \\
\hline
\end{tabular}

Fuente: elaboración propia

Pvalor $=0,019<0,05$. Se rechaza la hipótesis de independencia entre las variables: la opinión de los administrativos es dependiente del tipo de cargo que este ocupe.

Tabla 47. Libertad del personal para desarrollar el trabajo

\begin{tabular}{|c|l|c|c|c|}
\cline { 3 - 5 } \multicolumn{2}{c|}{} & Frecuencia & $\%$ & \% acumulado \\
\hline \multirow{4}{*}{ Opciones de respuesta } & Nunca & 13 & 4,4 & 4,4 \\
\cline { 2 - 5 } & Rara vez & 76 & 25,6 & 30,0 \\
\cline { 2 - 5 } & Usualmente & 115 & 38,7 & 100,0 \\
\cline { 2 - 5 } & Siempre & 93 & 31,3 & 61,3 \\
\cline { 2 - 5 } & Total & 297 & 100,0 & \\
\hline
\end{tabular}

Fuente: elaboración propia

Tabla 48. Libertad del personal para desarrollar el trabajo según cargo ocupado

\begin{tabular}{|c|l|c|c|c|c|c|}
\cline { 3 - 7 } \multicolumn{2}{c|}{} & Nunca & Rara vez & Siempre & Usualmente & Total \\
\hline \multirow{4}{*}{ Cargo } & Auxiliar & 0 & 7 & 12 & 15 & 34 \\
\cline { 2 - 7 } & Jefe & 2 & 6 & 9 & 6 & 23 \\
\cline { 2 - 7 } & Profesional & 7 & 34 & 33 & 41 & 115 \\
\cline { 2 - 7 } & Secretario & 1 & 8 & 14 & 17 & 40 \\
\cline { 2 - 7 } & Técnico & 3 & 21 & 25 & 36 & 85 \\
\hline \multirow{7}{*}{ Total } & 13 & 76 & 93 & 115 & 297 \\
\hline
\end{tabular}




\begin{tabular}{|l|c|c|c|}
\hline \multicolumn{1}{|c|}{ Prueba de independencia } & Valor & Gl & Sig. asintótica (bilateral) \\
\hline Chi-cuadrado de Pearson & $8,322^{\mathrm{a}}$ & 12 & 0,759 \\
\hline Razón de verosimilitudes & 9,675 & 12 & 0,644 \\
\hline N. ${ }^{\circ}$ de casos válidos & 297 & & \\
\hline
\end{tabular}

Fuente: elaboración propia

Pvalor $=0,759>0,05$. Se acepta la hipótesis de independencia entre las variables: la opinión de los administrativos es independiente del tipo de cargo que este ocupe.

Tabla 49. El jefe evita que usted sugiera cambios, aunque sean necesarios

\begin{tabular}{|c|l|c|c|c|}
\cline { 3 - 5 } \multicolumn{2}{c|}{} & Frecuencia & $\%$ & \% acumulado \\
\hline \multirow{3}{*}{ Opciones de respuesta } & Nunca & 76 & 25,6 & 25,6 \\
\cline { 2 - 5 } & Rara vez & 122 & 41,1 & 66,7 \\
\cline { 2 - 5 } & Usualmente & 71 & 23,9 & 100,0 \\
\cline { 2 - 5 } & Siempre & 28 & 9,4 & 76,1 \\
\cline { 2 - 5 } & Total & 297 & 100,0 & \\
\hline
\end{tabular}

Fuente: elaboración propia

Tabla 50. El jefe evita que usted sugiera cambios, aunque sean necesarios según cargo ocupado

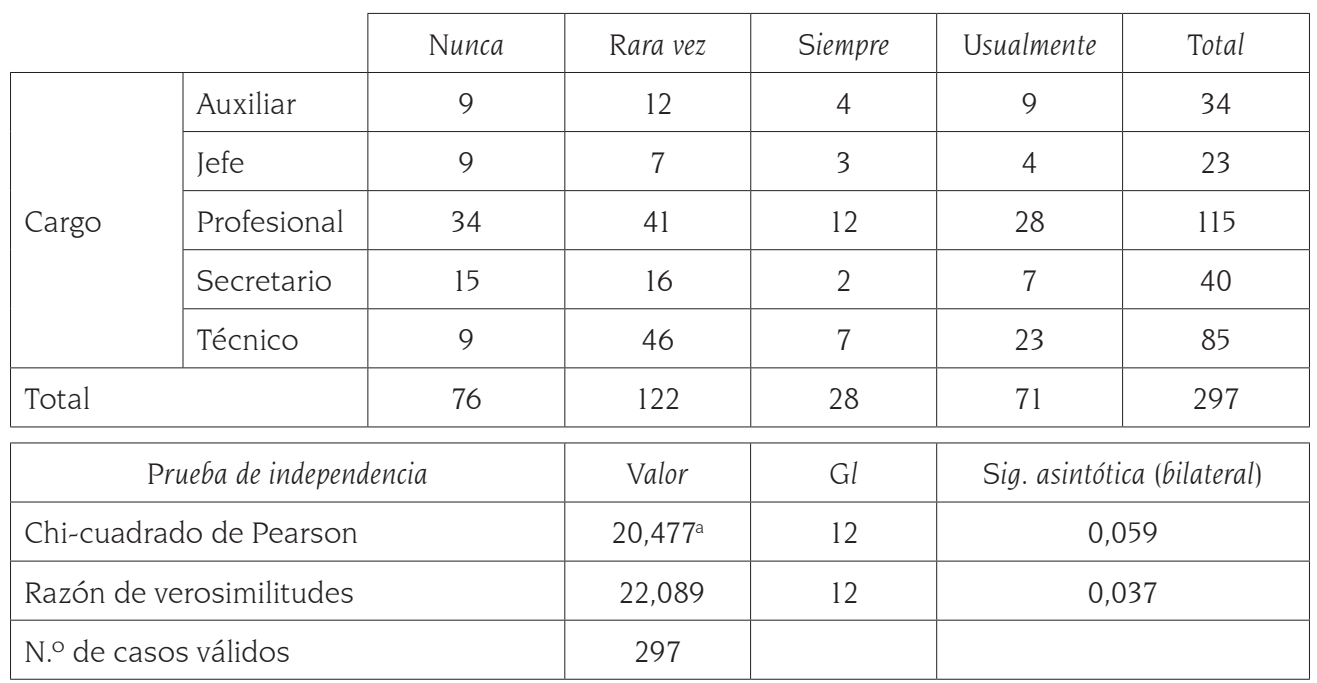

Fuente: elaboración propia

Pvalor $=0,059>0,05$. Se acepta la hipótesis de independencia entre las variables: la opinión de los administrativos es independiente del tipo de cargo que este ocupe. 
Felicidad organizacional: mediación entre la valoración del empleado y la productividad. Una mirada ...

Tabla 51. El jefe valora las nuevas ideas, procesos o sugerencias, presentadas por el personal

\begin{tabular}{|c|l|c|c|c|}
\cline { 3 - 5 } \multicolumn{2}{c|}{} & Frecuencia & $\%$ & \% acumulado \\
\cline { 2 - 5 } & Nunca & 10 & 3,4 & 3,4 \\
\cline { 2 - 5 } Opciones de respuesta & Rara vez & 42 & 14,1 & 17,5 \\
\cline { 2 - 5 } & Usualmente & 146 & 49,2 & 100,0 \\
\cline { 2 - 5 } & Siempre & 99 & 33,3 & 50,8 \\
\cline { 2 - 5 } & Total & 297 & 100,0 & \\
\hline
\end{tabular}

Fuente: elaboración propia

Tabla 52. El jefe valora las nuevas ideas, procesos o sugerencias, presentadas por el personal según cargo ocupado

\begin{tabular}{|l|l|c|c|c|c|c|}
\cline { 3 - 7 } \multicolumn{2}{c|}{} & Nunca & Rara vez & Siempre & Usualmente & Total \\
\hline \multirow{4}{*}{ Cargo } & Auxiliar & 3 & 2 & 16 & 13 & 34 \\
\cline { 2 - 7 } & Jefe & 0 & 1 & 13 & 9 & 23 \\
\cline { 2 - 7 } & Profesional & 3 & 20 & 34 & 58 & 115 \\
\cline { 2 - 7 } & Secretario & 2 & 4 & 11 & 23 & 40 \\
\cline { 2 - 7 } & Técnico & 2 & 15 & 25 & 43 & 85 \\
\hline \multirow{4}{*}{ Total } & 37 & 101 & 44 & 115 & 297 \\
\hline
\end{tabular}

\begin{tabular}{|l|c|c|c|}
\hline \multicolumn{1}{|c|}{ Prueba de independencia } & Valor & Gl & Sig. asintótica (bilateral) \\
\hline Chi-cuadrado de Pearson & $18,694^{\text {a }}$ & 12 & 0,096 \\
\hline Razón de verosimilitudes & 18,803 & 12 & 0,093 \\
\hline N. $^{\circ}$ de casos válidos & 297 & & \\
\hline
\end{tabular}

Fuente: elaboración propia

Pvalor $=0,096>0,05$. Se acepta la hipótesis de independencia entre las variables: la opinión de los administrativos es independiente del tipo de cargo que este ocupe.

Tabla 53. La organización fomenta acciones para promover su innovación

\begin{tabular}{|c|l|c|c|c|}
\cline { 3 - 5 } \multicolumn{2}{c|}{} & Frecuencia & $\%$ & \% acumulado \\
\hline \multirow{4}{*}{ Opciones de respuesta } & Nunca & 37 & 12,5 & 12,5 \\
\cline { 2 - 5 } & Rara vez & 101 & 34,0 & 46,5 \\
\cline { 2 - 5 } & Usualmente & 115 & 38,7 & 100,0 \\
\cline { 2 - 5 } & Siempre & 44 & 14,8 & 61,3 \\
\cline { 2 - 5 } & Total & 297 & 100,0 & \\
\hline
\end{tabular}

Fuente: elaboración propia 
Tabla 54. La organización fomenta acciones para promover su innovación según cargo ocupado

\begin{tabular}{|c|l|c|c|c|c|c|}
\cline { 3 - 7 } \multicolumn{2}{c|}{} & Nunca & Rara vez & Siempre & Usualmente & Total \\
\hline \multirow{4}{*}{ Cargo } & Auxiliar & 10 & 11 & 4 & 9 & 34 \\
\cline { 2 - 7 } & Jefe & 0 & 4 & 9 & 10 & 23 \\
\cline { 2 - 7 } & Profesional & 14 & 44 & 10 & 47 & 115 \\
\cline { 2 - 7 } & Secretario & 4 & 14 & 4 & 18 & 40 \\
\cline { 2 - 7 } & Técnico & 9 & 28 & 17 & 31 & 85 \\
\hline \multirow{7}{*}{ Total } & 37 & 101 & 44 & 115 & 297 \\
\hline
\end{tabular}

\begin{tabular}{|l|c|c|c|}
\hline \multicolumn{1}{|c|}{ Prueba de independencia } & Valor & Gl & Sig. asintótica (bilateral) \\
\hline Chi-cuadrado de Pearson & $30,272^{\mathrm{a}}$ & 12 &, 003 \\
\hline Razón de verosimilitudes & 29,074 & 12 &, 004 \\
\hline N. $^{\circ}$ de casos válidos & 297 & & \\
\hline
\end{tabular}

Fuente: elaboración propia

Pvalor $=0,096>0,05$. Se acepta la hipótesis de independencia entre las variables: la opinión de los administrativos es independiente del tipo de cargo que este ocupe.

\section{Conclusiones}

En sentido crítico, el capítulo integra el abordaje cualitativo del fenómeno de la felicidad organizacional en una institución educativa de Montería, Colombia, a partir de los hallazgos cuantitativos encontrados por medio de los procedimientos estadísticos aplicados. Este apartado es el resultado de la triangulación de los instrumentos, cuyo proceso permitió identificar algunas contradicciones entre los resultados de la encuesta respecto a las apreciaciones obtenidas a través de la observación no participante y de la revisión documental, lo que evidencia fortalezas en el trabajo en equipo y compromiso con la organización/función y debilidades en la satisfacción laboral e innovación.

Por consiguiente, se fundamentan las conclusiones desde la relación existente entre la opinión de administrativos y el cargo que desempeñan.

\section{Trabajo en equipo}

En cuanto a la variable trabajo en equipo, la cual establece la capacidad intelectual, el respeto y la colaboración como componentes que aportan a su desarrollo, se pudo concluir que hay una relación directa de esta variable con la felicidad organizacional. El estado actual de la variable demuestra un nivel satisfactorio, que es fundamental para transformar posibilidades en soluciones que contribuyan a la sostenibilidad de la universidad.

- En lo que concierne a capacidades intelectuales existe correspondencia entre las compe- 
tencias profesionales de los administrativos y las habilidades que se requieren para el ejercicio de la función. La institución cuenta con una estructura organizacional que establece los cargos, perfiles y funciones, lo cual fortalece el desarrollo de la dimensión a través de la asignación de funciones acordes a los conocimientos, aprovechando las capacidades personales y promoviendo las relaciones para generar un ambiente de confianza, motivación y respeto. Asimismo, hay correspondencia entre el cargo que desempeñan y la alta tramitología en las funciones, lo que se asocia con la organización y planificación de procesos, la claridad en cuanto a la asignación de las tareas y el aprovechamiento de las potencialidades de los trabajadores. Además, el jefe valora las opiniones, ideas y sugerencias del equipo de trabajo para la toma de decisiones y se preocupa por crear un clima de cooperación. Finalmente, se infiere que el aprovechamiento de las capacidades intelectuales aporta al rendimiento de los administrativos.

- De acuerdo al componente respeto, se evidencia la relación existente entre la posición del funcionario en la institución y el nivel de consideración y valoración social del individuo en el contexto organizacional. Se evidencia tolerancia a las diferencias, lo que contribuye al trabajo en equipo. En la universidad se promueve el valor del respeto, se fomentan acciones para el fortalecimiento de las relaciones entre compañeros y no predomina el conflicto al momento de interactuar entre ellos y los jefes. Finalmente, se infiere que el respeto como valor institucional, en el marco del trabajo en equipo, conduce a identificar y potencializar las habilidades para las relaciones personales con los compañeros a partir del reconocimiento social de cada colaborador, lo cual aporta al desarrollo de las funciones.

- En cuanto a colaboración se pudo establecer entre los administrativos la disposición a aceptar y proporcionar apoyo dentro de los procesos, dirigir esfuerzos hacia un objetivo común y sostener relaciones coordinadas y efectivas entre las diferentes dependencias. En la institución se estimulan la participación y el trabajo colectivo, uniendo capacidades y talentos desde los distintos cargos hacia un objetivo común. Este valor facilita la articulación de las capacidades profesionales de cada persona y las habilidades que se requieren para el ejercicio de la función, contribuyendo al buen funcionamiento del trabajo en equipo como dimensión en la felicidad organizacional.

\section{Satisfacción laboral}

En cuanto a la variable satisfacción laboral, la cual establece las relaciones personales, experiencia y expectativas como componentes que aportan en su desarrollo, se pudo concluir que hay una relación directa de esta variable con la felicidad organizacional. El estado actual de la variable en los administrativos de la universidad se encuentra en una perspectiva poco favorable, lo cual dificulta la capacidad de contribuir al logro de los objetivos corporativos:

- En lo que respecta a experiencia, se identificó que el conjunto de conocimientos adquiridos y aplicados al trabajo son valorados por la institución, pues los administrativos 
cuentan con los recursos necesarios para la realización de las funciones. No obstante, a pesar de recibir de manera oportuna su salario los funcionarios manifiestan que dicha remuneración se basa más en las relaciones interpersonales con los directivos y mandos altos que en los méritos y resultados del trabajo, especialmente en la sede de Lorica.

- En cuanto a la expectativa, se encontró que la institución no se interesa por contribuir al desarrollo personal, familiar y profesional de los administrativos. Los aportes en relación al mejoramiento del bienestar laboral no se toman en cuenta, lo que disminuye el interés por presentar resultados de calidad y limitando el aprovechamiento de las habilidades para desarrollar competencias de manera integral, todo ello influye en el clima laboral y en la proyección de la organización.

- En cuanto a las relaciones personales, aunque se establecen redes comunicativas para el fortalecimiento del diálogo y la participación entre compañeros, dichas acciones no se encuentran fundamentadas en la estimulación del valor de la felicidad. Para los administrativos, se toman más en cuenta las relaciones personales que los resul tados, lo cual afecta la forma de colaboración, la transformación de las emociones e intereses a mediano y largo plazo, impactando directamente en la sostenibilidad de la universidad.

Desde la dimensión satisfacción laboral no existe concordancia entre las experiencias y las expectativas de los administrativos con respecto al trabajo, lo que genera un desequilibrio entre el esfuerzo individual y colectivo. En este sentido, las expectativas se ven afectadas por la falta de redes comunicativas cimentadas en la valoración individual que presenta la organización hacia sus funcionarios.

\section{Compromiso con la función y organización}

En cuanto a la dimensión compromiso con la función y organización, la cual aborda aspectos de tipo emocional, de pertenencia y normatividad en su desarrollo, se pudo concluir que hay una relación directa de esta variable con la felicidad organizacional. El estado actual de esta variable en términos generales es positivo, sin embargo, algunos resultados son contradictorios al contrastar los obtenidos con la encuesta y con la observación no participante:

- Desde el compromiso emocional se evidencia la falta de identificación de los trabajadores con la institución, motivada en gran medida, porque las expectativas individuales están en disonancia con los intereses institucionales. Sin embargo, independientemente de las expectativas individuales, los trabajadores reconocen la importancia de la combinación de esfuerzos entre los diferentes miembros de las áreas para aportar al desarrollo de la organización.

- De acuerdo al compromiso de pertenencia, los administrativos asocian el grado de conformidad y aceptación de la remuneración salarial, con el pago a tiempo y el monto 
que reciben por el trabajo que realizan. No obstante, al realizar las comparaciones de ingresos por cargos, consideran que el monto es muy desigual entre pares y distante a las funciones asignadas y a los resultados obtenidos. Es notoria la inequidad en cuanto al cargo y la función y la poca valoración de la organización, lo cual se refleja en la permanencia en el puesto de trabajo a pesar de la desmotivación, principalmente en la sede de Lorica.

- En lo que respecta al compromiso normativo, la consolidación de las normas/lineamientos que benefician a la empresa y representan los intereses de los trabajadores, influye en el adecuado cumplimiento de las tareas más básicas. En la universidad, los administrativos cumplen las funciones y respetan las directrices que conllevan a la asignación de tareas.

En un significativo porcentaje, los funcionarios no tienen claras las políticas y lineamientos organizacionales, existe desconocimiento de la información institucional, lo que influye negativamente en el óptimo funcionamiento de los procesos entre las distintas dependencias. A pesar de que el correo corporativo es considerado el principal medio por donde circula la información, el personal poco lo usa y aprovecha. Hay ausentismo en los puestos de trabajo, lo que influye en el desconocimiento de lo que pasa a diario y de los nuevos lineamientos que se formalizan, la falta de claridad con respecto a los procesos, los retrasos en el cumplimiento de las tareas asignadas, el poco dinamismo entre las áreas y la poca calidad de los resultados individuales y colectivos.

\section{Innovación}

En cuanto a la variable innovación, la cual se relaciona con la cultura organizacional, motivación y creatividad, se pudo concluir que es escasamente estimulada en la universidad, lo que afecta la capacidad de desarrollar servicios y procesos de manera novedosa, eficiente, productiva y orientada a la proyección de la organización en el contexto, lo que finalmente no la convierte en una ventaja competitiva para mantenerse vigente en el mercado regional y nacional; más aún cuando algunos resultados son contradictorios al contrastarlos con la encuesta y la observación no participante:

- Desde la cultura organizacional, los administrativos reconocen que las condiciones que brinda el medio laboral no posibilitan el surgimiento de las ideas creativas, no es común el estímulo a la capacidad de idear o crear algo desde la novedad y originalidad que aporte al desarrollo de las funciones, de los procesos y de la organización. Es limitado el interés de los funcionarios por conocer el contexto cultural en el que se desenvuelve la institución, lo que impide el diseño e implementación de estrategias que surjan a partir de nuevos y mejorados procesos/servicios/productos que el medio necesite, reconozca y valore por su aporte, es decir, aquellos cambios que la universidad construya, que se visibilicen externamente y que el mercado experimente y aproveche.

- En cuanto a motivación, se evidencia la falta de incentivos al empleado para arriesgarse 
a proponer ideas y desarrollarlas. En la actualidad, el trabajador se limita a cumplir con lo que se asigna, lo que disminuye el surgimiento de nuevos procesos y protocolos que incidan directamente en la satisfacción laboral, el trabajo en equipo, el compromiso con la función y la organización, en la innovación y, por ende, en la productividad. Lo anterior se traduce en la resistencia a probar nuevos métodos para la realización de las funciones dado que significa para algunos jefes pérdida de tiempo productivo y para los funcionarios desgaste al no ser valorado este trabajo. Al no ser motivados y motivadores, los funcionarios no participan activamente en los procesos, no son autónomos ni originales, no varían las formas de trabajar y no descubren nuevas formas de cumplir funciones.

- La creatividad se encuentra escasamente vinculada a la jornada laboral. Teniendo en cuenta que el componente depende del cargo, los trabajadores expresaron no contar con las mismas oportunidades para desarrollar habilidades creativas, pues las funciones en las áreas se desarrollan de distintas maneras. Además, reconocieron que promover, al interior de la institución, políticas y lineamientos corporativos que abonen el camino hacia la creatividad e innovación, como proceso de aprendizaje constante, se convierte en un aporte colectivo gestado desde los empleados hacia la institución. Las características particulares de la universidad exigen procesos de adaptación a las nuevas situaciones y factores que se generan dentro y desde el entorno, los cuales son el insumo para los procesos creativos. Es importante mencionar que los administrativos destacaron la importancia de incentivar la innovación entre los empleados, para esto, la institución debe abandonar algunas normas obsoletas en cuanto a la gestión del clima organizacional y al impacto de la felicidad en la productividad, a los conocimientos desactualizados para el desarrollo de las tareas y a las experiencias poco novedosas al servicio de la institución.

Finalmente, aunque el área de talento humano de la universidad informó la existencia de un estudio adelantado sobre el tema, se comprobó que solo se tiene registrada una monografía (Jiménez y Galarcio, 2014), que obedece más a un producto para alcanzar un título de especialización que a un estudio organizacional que haya servido de insumo para la toma de decisiones.

\section{Referencias}

Achor, S. (2010). The Happiness advantage: The Seven Principles of Positive Psychology That Fuel Success and Performance at Work. Nueva York: Virgin Digital Publishing.

Baker, W. y Sinkula, J. (1999). Learning orientation, market orientation, and innovation: integrating and extending models of organizational performance (Vol. 27). Boston: Journal of the Academy of Marketing Science.

Betancourt, A. (1985). Organizaciones y administración: un enfoque de sistemas. Cali: Norma.

Carballeira, M. González, J. Marrero, R. (2015). Diferencias transculturales en bienestar subjetivo: México y España. Anales de Psicología, 31(1), 199-206. https://doi.org/10.6018/analesps.31.1.166931 
Cardona, C. (2002). Fundamentos de administración. Bogotá: ECOE.

Dankhe, G. (1986). Investigación y comunicación. En C. Fernández-Collado y G.L. Dankhe (Eds.), La comunicación humana: ciencia social (pp. 385-454). Ciudad de México: Mc.Graw-Hill.

Drucker, P. (1993). La sociedad poscapitalista. Buenos Aires: Sudamericana.

Dutschke, G. (2013). Factores condicionantes de felicidad organizacional. Estudio exploratorio de la realidad en Portugal. Revista de Estudios Empresariales, (1), 21-43.

Fernández, I. (2015). Felicidad organizacional: cómo construir felicidad en el trabajo. Santiago de Chile: Ediciones B.

Fisher, C. (2010). Happiness at Work. International Journal of Management Reviews, 12(4), 384-412.

Gutiérrez, M. y Gonçalves, T. (2013). Activos para el desarrollo, ajuste escolar y bienestar subjetivo de los adolescentes. International Journal of Psychology and Psychological Therapy, 13(3), 339-355.

Hernández Sampieri, R., Fernández, C. y Baptista, P. (2006). Metodología de la Investigación (4ª ed.). México: McGraw-Hill.

Hosie, P., Cooper, C. y Sevastos, P. (2007). The Happy Productive Worker Thesis and Australian Managers. Journal of Human Values 13(2), 151-176.

Malluk, A. y Bedoya, S. (2016). Diagnóstico de comunicación organizacional de la institución educativa Cristobal Colón de Montería,(tesis de pregrado) Universidad Pontificia Bolivariana, Monteria, Colombia.

Nader, M. Castro, A. (2010). Teorías implícitas del liderazgo, LMX y bienestar laboral: generalización de un modelo teórico. Revista de Psicología, 28(2), 227-258.

Meyer, J. y Allen, N. (1984). Testing the side-bet theory of organization commitment: Some methodological considerations. Journal of Applied Psychology, 69(3), 372-378.

Gamboa, E. (2006). Satisfacción laboral: descripción teórica de sus determinantes. Recuperado de http://www.psicologiacientifica.com/satisfaccion-laboral-determinantes

Oberle, E., Schonert-Reichl, K. y Zumbo, B. (2011). Life satisfaction in early adolescence: Personal, neighborhood, school, family, and peer influences. Journal of Youth and Adolescence, 40(7), 889-901.

Corral, S. y Pereña, J. (2003). Cuestionario de Clima Laboral (CLA): test para evaluar el clima laboral de las empresas y las organizaciones. Madrid: TEA.

Pulido, F. y Herrera, F. (2018). Predictores de la felicidad y la inteligencia emocional

en la educación secundaria. Revista Colombiana de Psicología, 27(1), 71-84. https://doi.org/10.15446/rcp. v27n1.62705

Quiceno, J. y Vinaccia, S. (2015). Calidad de vida, fortalezas personales, depresión y estrés en adolescentes según sexo y estrato. International Journal of Psychology and Psychological Therapy, 14(2), 155-170.

Revista Líderes. (2014). Las empresas miran en la felicidad un nuevo indicador. Recuperado de https:// www.revistalideres.ec/lideres/empresas-miran-felicidad-nuevo-indicador.html

Rodríguez, M. (2010). Métodos de investigación: diseño de proyectos y desarrollo de tesis en ciencias administrativas, organizacionales y sociales. México: Universidad Autónoma de Sinaloa. 
Caycho Rodríguez, C. (2010). Variables psicológicas asociadas con la felicidad en centros periurbanos y urbanos marginales de Lima. UCV-Scientia, 2(1), 61-68

Rodríguez, C. y Caño, A. (2012). Autoestima en la adolescencia: análisis y estrategias de intervención. International Journal of Psychology and Psychological Therapy, 12(3), 389-403.

Salanova, M., Martínez, I. y Llorens, S. (2005). Psicología organizacional positiva. en F. Palací (Coord.), Psicología de la organización (pp. 349-376). Madrid: Prentice-Hall.

Salanova, M. Martínez, I. Llorens, S. (2014). Una mirada más "positiva" a la salud ocupacional desde la Psicología Organizacional Positiva en tiempos de crisis: aportaciones desde el equipo de investigación WONT. Papeles del Psicólogo, 35(1), 22-30.

Salessi, S. y Omar, A. (2017). Satisfacción laboral: un modelo explicativo basado en variables disposicionales. Revista Colombiana de Psicología, 26(2), 329-345. doi: 10.15446/rcp.v26n2.60651

Särndal, C. Swensson, B. y Wretman, J. (2003). Model assisted survey sampling. Nueva York: Springer Science \& Business Media.

Seligman, M. (1999). The president’s address. American Psychologist, 54, 559-562.

Seligman, M. y Csikszentmihalyi, M. (2000). Positive psychology: An introduction. American Psychologist, 55, 5-14.

Shona, L. Brown, M. Eisenhardt, K. (1997). The Art of Continuous Change: Linking Complexity Theory and TimePaced Evolution in Relentlessly Shifting Organizations (Vol. 42). Boston: Administrative Science Quarterly.

Stake, R. (2007). Investigación con estudio de casos (4a ed.). Madrid: Morata.

Tarragona, M. (2013). Psicología Positiva y Terapias Constructivas: Una Propuesta Integradora. Terapia Psicológica, 31(1), 115125.

Oswald, A., Proto, E. y Sgroi, D. (2015) Happiness and productivity. Journal of Labor Economics, 33(4), 789-822.

Yin, R. (1993). Applications of Case Study Research (Applied Social Research Methods Series, Vol. 34). Washington: Cosmos Corporation. 